\title{
Cahn-Hilliard stochastic equation: existence of the solution and of its density
}

\author{
CAROLINE CARDON-WEBER \\ Laboratoire de Probabilités et Modèles Aléatoires, CNRS-UMR 7599, Université Paris 6, 4 place \\ Jussieu,Tour 56,F-75252 Paris Cedex05.E-mail: weber@ccr.jussieu.fr
}

We show the existence and uniqueness of a function-valued process solution to the stochastic CahnHilliard equation driven by space-time white noise with a nonlinear diffusion coefficient. Then we show that the solution is locally differentiable in the sense of the Malliavin calculus, and, under some non-degeneracy condition on the diffusion coefficient, that the law of the solution is absolutely continuous with respect to Lebesgue measure.

Keywords: Cahn-Hilliard equation; Green function; Malliavin calculus; stochastic partial differential equations

\section{Introduction}

Set $D=[0, \pi]^{d}$, let $W$ denote a one-dimensional $(d+1)$-parameter Wiener process, and consider the stochastic partial differential equation (SPDE)

$$
\frac{\mathrm{d} u}{\mathrm{~d} t}+\left(\Delta^{2} u-\Delta f(u)\right)=\sigma(u) \dot{W}
$$

with initial condition $u(0, \cdot)=u_{0}$ and homogeneous Neumann boundary conditions

$$
\frac{\partial u}{\partial n}=\frac{\partial \Delta u}{\partial n}=0 \text { on } \partial D
$$

This is a stochastic version of the Cahn-Hilliard equation $(\sigma=0)$. This equation describes the complicated phase separation and coarsening phenomena in a melted alloy that is quenched to a temperature at which only two different concentration phases can exist stably. $u$ represents a scaled concentration, and $-\Delta u+f(u)$ represents the chemical potential. The Neumann boundary conditions reflect the conservation of mass and insulation from outside. The function $f$ is the derivative of the homogeneous free energy $F$. In its original form, $F$ contains a logarithmic term. In some cases, $F$ can be approximated by an even-degree polynomial with positive dominant coefficient. For more physical background on this equation, see, for example, Cahn and Hilliard (1958) and Novick-Cohen and Segel (1984).

The existence and uniqueness of the solution to (1.1) have already been proved by Debussche and Dettori (1995) when $f$ is the derivative of a logarithmic term in the case of logarithmic free energy and $\sigma=0$, and by Da Prato and Debussche (1996) in the case of an additive noise $(\sigma=1)$, when $f$ is a polynomial of odd degree in a set of distributions, and if 
$d \geqslant 2$ in the set of $L^{2}(D)$ functions only for Gaussian perturbations more regular than the white noise $W$. Our study is restricted to dimension $d$, where $d \in\{1,2,3\}$, on the domain $D=[0, \pi]^{d}$. We assume that $f$ is a polynomial of degree 3 . To prove existence and uniqueness of an almost surely (a.s.) continuous solution, we need some information on the Green function $G$ of the operator $\partial / \partial t+\Delta^{2}$. This operator has a well-defined expression as a Fourier series, but this is not easy to use. We need other estimates of $G$; for this, we use a result from Eidelman and Ivasisen (1970) on Green's functions on a smooth domain. We have extended these estimates from smooth domains to the domain $D$ in the Appendix. In the first part of this paper we use the estimate of the Green function to prove first the existence and uniqueness of the solution for a similar equation with truncated coefficients. This yields the existence and uniqueness of the solution on the time interval $[0, \tau[$, where $\tau$ is a stopping time. To prove that $\tau=+\infty$, we need a priori estimates in the space $L^{\infty}\left([0, T] ; L^{q}(D)\right)$, $q \geqslant 4$. For this we prove two different upper estimates and use an interpolation method. In these computations we need the degree of $f$ not to be larger than 3. The approach is similar to that used by Gyöngy (1998) and Da Prato and Gatarek (1995) for another nonlinear parabolic stochastic equation with correlated noise, the Burgers stochastic equation. We then study the regularity of $u$ and prove that if $u_{0}$ is Hölder continuous, then $u$ also has Hölder regular trajectories. More precisely, in the case $d=1$, if $u_{0}$ is differentiable $\left(u_{0} \in \mathscr{C}^{2}(D)\right)$, the solution $u$ is differentiable with respect to the space variable.

In the second part of this paper, we study the existence of the density of $u(t, x)$ for $t>0$ and $x \in] 0, \pi\left[{ }^{d}\right.$. For this we use the Malliavin calculus (see Nualart 1995) associated with the space-time white noise $W$. Similar results have been obtained by Morien (1999) in the case of the stochastic Burgers equation. Concerning the Burgers equation, let us mention the work of Lanjri Zaidi and Nualart (1999), which proves the existence of a density under weaker conditions. This refinement seems to be more difficult to obtain in our case, because we do not have good lower estimates of the Green function.

The paper is organized as follows. We first state the hypotheses and the main results of this paper; then we give some lemmas on the Green function $G$. In Section 2, we establish the existence of the solution to the SPDE (1.1) and study its regularity. In Section 3, we prove the absolute continuity of the solution $u(t, x)$ for $t>0$ and $x \in] 0, \pi\left[{ }^{d}\right.$. Finally, the basic pointwise upper estimates of the Green function and of its derivatives are shown in the Appendix. As usual, constants $C$ and $c$ may change from one line to the next; we denote by $C_{\alpha}$ a constant which depends on some parameter $\alpha$. We denote the space variable by $x$ and the space integral by $\int \ldots \mathrm{d} x$, even if the dimension $d$ is not 1 , and denote by $\|\cdot\|_{q}$ the $L^{q}$-norm with respect to $\mathrm{d} x$.

\subsection{Hypotheses and results}

We make the following assumptions:

Assumption 1. $f$ is a polynomial of degree 3 with positive dominant coefficient.

Assumption 2. $\sigma: \mathbb{R} \mapsto \mathbb{R}$ is a bounded and Lipschitz function. 
Assumption 3. $u_{0}$ belongs to $L^{q}(D)$ for $q \geqslant 4$.

Assumption 4. $u_{0}$ is a continuous function on D.

Assumption $4^{\prime} . u_{0}$ is an $\alpha$-Hölder continuous function on $\left.D, \alpha \in\right] 0,1[$.

Assumption 4". $d=1$ and $u_{0}$ is an $\alpha$-Hölder continuous function on $D, \alpha \geqslant 2$; moreover, $u_{0}^{\prime}(0)=u_{0}^{\prime}(\pi)=0$.

Assumption 5. The function $\sigma$ does not vanish $(\sigma \neq 0)$.

Remark 1.1. In Assumption 1, we have assumed that $f$ is of degree 3; the case of degree 1 is easier and its proof is omitted.

We will use Assumptions 4, 4' and 4" only in Sections 2.3 and 3 to prove the regularity property of the solution, and Assumption 5 only in Section 3.2 to obtain the existence of a density.

We suppose that $W=\{W(t, x), t \in[0, T] ; x \in D\}$ is a one-dimensional, $(d+1)$ parameter Wiener process on the probability space $(\Omega, \mathscr{F}, P)$; as usual, we set $\mathscr{F}_{t}=$ $\sigma(W(s, x) ; s \leqslant t, x \in D)$.

Let $A$ denote the operator $-\Delta$ on the domain $\mathscr{D}(A)=\left\{u \in H^{2}(D) ; \partial u / \partial n=0\right.$ on $\left.\partial D\right\}$. The following family $\left(\varepsilon_{k}\right)_{k \in \mathbb{N}^{d}}$ is a basis of eigenfunctions of $A$ in $L^{2}(D)$. If $d=1$,

$$
\begin{aligned}
& \varepsilon_{k}(x)=\cos (k x) \sqrt{\frac{2}{\pi}} \text { if } k \neq 0, \\
& \varepsilon_{0}(x)=\frac{1}{\sqrt{\pi}}
\end{aligned}
$$

and for $d \in\{2,3\}$,

$$
\varepsilon_{k}(x)=\prod_{i=1}^{d} \varepsilon_{k_{i}}\left(x_{i}\right)
$$

associated with the eigenvalues $\lambda_{k}=\sum_{i=1}^{d} k_{i}^{2}=|k|^{2}$. By convention, denote by $\mathbb{N}^{d, *}$ the set $\mathbb{N}^{d} \backslash\{0\}$. The semigroup $S(t)$ generated by $-A^{2}$ is denoted by $S(t)=\mathrm{e}^{-t A^{2}}$, that is, for $z \in L^{2}(D)$

$$
S(t) z=\sum_{k \in \mathbb{N}^{d}} \mathrm{e}^{-\lambda_{k}^{2} t}\left\langle z, \varepsilon_{k}\right\rangle \varepsilon_{k},
$$

where $\langle\cdot, \cdot\rangle$ denotes the usual scalar product in $L^{2}(D)$; this is a convolution semigroup with the Green function $G$ defined by

$$
G(t, x, y)=\sum_{k \in \mathbb{N}^{d}} \mathrm{e}^{-\lambda_{k}^{2} t} \varepsilon_{k}(x) \varepsilon_{k}(y) .
$$

The following lemma is proved in the Appendix. 
Lemma 1.2. There exist $C>0$ and $c>0$ such that, for $t \in] 0, T], x, y \in D, \alpha a d$ dimensional exponent satisfying $|\alpha| \leqslant 3$ :

$$
\begin{gathered}
|G(t, x, y)| \leqslant \frac{C}{t^{d / 4}} \exp \left(-c \frac{|x-y|^{4 / 3}}{|t|^{1 / 3}}\right), \\
\left|\partial_{x}^{\alpha} G(t, x, y)\right| \leqslant \frac{C}{t^{(d+|\alpha|) / 4}} \exp \left(-c \frac{|x-y|^{4 / 3}}{|t|^{1 / 3}}\right), \\
\left|\partial_{t} G(t, x, y)\right| \leqslant \frac{C}{t^{(d+4) / 4}} \exp \left(-c \frac{\mid x-y^{4 / 3}}{|t|^{1 / 3}}\right) .
\end{gathered}
$$

We wish to prove the existence and uniqueness of the solution to the SPDE (1.1). Since the derivative of $W$ is formal, this can be made rigorous only in a weak sense, as in Walsh (1986) for the stochastic heat equation. We say that $u$ is a weak solution of (1.10) if, for each $\phi \in C^{4}(D)$ such that $\partial \phi / \partial n=\partial \Delta \phi / \partial n=0$ on $\partial D$, $u$ satisfies

$$
\begin{aligned}
\int_{D}\left(u(t, x)-u_{0}(x)\right) \phi(x) \mathrm{d} x= & -\int_{0}^{t} \int_{D} \Delta^{2} \phi(x) u(s, x) \mathrm{d} x \mathrm{~d} s+\int_{0}^{t} \int_{D} \Delta \phi(x) f(u(s, x)) \mathrm{d} x \mathrm{~d} s \\
& +\int_{0}^{t} \int_{D} \phi(s, x) \sigma(u(s, x)) W(\mathrm{~d} x, \mathrm{~d} s) .
\end{aligned}
$$

As usual, $u$ is a solution to (1.9) if and only if it solves the following evolution equation:

$$
\begin{aligned}
u(t, x)= & \int_{D} G(t, x, y) u_{0}(y) \mathrm{d} y+\int_{0}^{t} \int_{D} \Delta G(t-s, x, y) f(u(s, y)) \mathrm{d} y \mathrm{~d} s \\
& +\int_{0}^{t} \int_{D} G(t-s, x, y) \sigma(u(s, y)) W(\mathrm{~d} y, \mathrm{~d} s)
\end{aligned}
$$

for $x \in D, t \in[0, T]$.

The main results of this paper are the following:

Theorem 1.3. Suppose that Assumptions 1-3 hold; there exists a unique process $u=$ $\{u(t, x), t \in[0, T], x \in D\}$ in $L^{\infty}\left([0, T], L^{q}(D)\right)$, that is adapted, that is to say, $u(t, x)$ is $\mathscr{F}_{t}$-measurable for $(t, x) \in[0, T] \times D$, and satisfies the evolution equation (1.10).

Theorem 1.4. If Assumptions 1-4 are satisfied, then the solution to (1.10) has a.s. continuous trajectories.

If the Assumptions 1-3 and $4^{\prime}$ are satisfied, then the trajectories of the solution to (1.10) are a.s. $\beta$-Hölder continuous in $t$ and $\beta^{\prime}$-Hölder continuous in $x$, with $\beta \leqslant \alpha / 4$, $\beta<\frac{1}{2}(1-d / 4)$ and $\beta^{\prime} \leqslant \alpha, \beta^{\prime}<(2-d / 2)$.

If $d=1$ and Assumptions $1-3$ and 4 (or $4^{\prime \prime}$ ) are satisfied, then the trajectories of the 
solution to (1.10) are a.s. $\beta$-Hölder continuous in $t$ and $\beta^{\prime}$-Hölder continuous in $x$ on $\left[t_{0}, T\right] \times D$ for $0<t_{0}<T($ or $[0, T] \times D)$, with $\beta<\frac{3}{8}$ and $\beta^{\prime}<\frac{3}{2}$.

Theorem 1.5. Suppose that Assumptions 1-4 and 5 are satisfied, and let $u$ denote the solution to (1.10). For $(t, x) \in] 0, T] \times] 0, \pi\left[{ }^{d}\right.$, the law of $u(t, x)$ is absolutely continuous with respect to the Lebesgue measure on $\mathbb{R}$.

\subsection{The Green function}

The following result, similar to Gyöngy (1998, Lemma 3.1), gives precise estimates of the regularizing effect of convolution with $G, \Delta G$ and $G^{2}$.

Lemma 1.6. Let $J$ be defined, for all $v \in L^{1}\left([0, T], L^{\rho}(D)\right), 0 \leqslant t_{0} \leqslant t \leqslant T$, and $x \in D$, by

$$
J(v)\left(t_{0}, t, x\right)=\int_{t_{0}}^{t} \int_{D} H(t-s, x, y) v(s, y) \mathrm{d} y \mathrm{~d} s .
$$

Then for any $\rho \in[1, \infty], q \in[\rho,+\infty]$, and $1 / r=1 / q-1 / \rho+1 \in[0,1], J$ is a bounded operator from $L^{1}\left([0, T], L^{\rho}(D)\right)$ to $L^{\infty}\left([0, T], L^{q}(D)\right)$ such that:

1. If $H(s, x, y)=G(s, x, y)$, there is a constant $C>0$ such that

$$
\left\|J(v)\left(t_{0}, t, \cdot\right)\right\|_{q} \leqslant C \int_{t_{0}}^{t}(t-s)^{(d / 4)(1 / r-1)}\|v(s, \cdot)\|_{\rho} \mathrm{d} s .
$$

2. If $H(s, x, y)=\Delta G(s, x, y)$ (if $d=3$, we also need $r<3$; and if $d=2, r \neq \infty)$, there is a constant $C>0$ such that

$$
\left\|J(v)\left(t_{0}, t, \cdot\right)\right\|_{q} \leqslant C \int_{t_{0}}^{t}(t-s)^{-(d+2) / 4+d /(4 r)}\|v(s, \cdot)\|_{\rho} \mathrm{d} s .
$$

3. If $H(s, x, y)=G^{2}(s, x, y)$ (if $d=3$, we also need $r<\frac{3}{2}$, and if $d=2, r \neq \infty$ ), there is a constant $C>0$ such that

$$
\left\|J(v)\left(t_{0}, t, \cdot\right)\right\|_{q} \leqslant C \int_{t_{0}}^{t}(t-s)^{-d / 2+d /(4 r)}\|v(s, \cdot)\|_{\rho} \mathrm{d} s .
$$

Proof. The proof is based on the inequalities of Minkowski and Young. We only prove (1.11); the proof of the two other inequalities is similar. Minkowski's inequality and Lemma 1.2 imply that

$$
\begin{aligned}
\left\|J(v)\left(t_{0}, t, \cdot\right)\right\|_{q} & \leqslant \int_{t_{0}}^{t}\left\|\int_{D} G(t-s, \cdot, y) v(s, y) \mathrm{d} y\right\|_{q} \mathrm{~d} s \\
& \leqslant c \int_{t_{0}}^{t}|t-s|^{-d / 4}\left\|\int_{D} \exp \left(-c \frac{|\cdot-y|^{4 / 3}}{|t-s|^{1 / 3}}\right)|v(s, y)| \mathrm{d} y\right\|_{q} \mathrm{~d} s .
\end{aligned}
$$


We observe that for $t>0$,

$$
\int_{\mathbb{R}^{d}} \exp \left(-\frac{c|x|^{4 / 3}}{t^{1 / 3}}\right) \mathrm{d} x=C t^{d / 4} .
$$

Let $r \geqslant 1$ be such that $1 / q+1=1 / r+1 / \rho$ ( $r$ exists because $1 \geqslant 1 / q+1-1 / \rho \geqslant 0)$. Young's inequality and (1.14) imply that

$$
\begin{aligned}
\left\|J(v)\left(t_{0}, t, \cdot\right)\right\|_{q} & \leqslant \int_{t_{0}}^{t}|t-s|^{-d / 4}\|v(s, \cdot)\|_{\rho}\left\|\exp \left(-c \frac{\left.|\cdot|\right|^{4 / 3}}{|t-s|^{1 / 3}}\right)\right\|_{r} \mathrm{~d} s \\
& \leqslant C \int_{t_{0}}^{t}|t-s|^{(d / 4)(1 / r-1)}\|v(s, \cdot)\|_{\rho} \mathrm{d} s .
\end{aligned}
$$

Note that, again using Young's inequality, the left-hand sides of (1.11)-(1.13) converge with the conditions which are made on $r$ when $d=2,3$.

The proof of following lemma, which is similar to the preceding one, will be omitted.

Lemma 1.7. Let $J_{\alpha}$ be defined, for all $v \in L^{1}\left([0, T], L^{\rho}(D)\right), 0 \leqslant t_{0} \leqslant t \leqslant T$, and $x \in D$, $\alpha \in] 0,1[$, by

$$
J_{\alpha}(v)\left(t_{0}, t, x\right)=\int_{t_{0}}^{t} \int_{D}(t-s)^{-\alpha} \Delta G(t-s, x, y) v(s, y) \mathrm{d} y \mathrm{~d} s .
$$

Then for any $\rho \in[1, \infty], q \in[\rho,+\infty]$, and $1 / r=1 / q-1 / \rho+1 \in[0,1], J$ is a bounded operator from $L^{1}\left([0, T], L^{\rho}(D)\right)$ to $L^{\infty}\left([0, T], L^{q}(D)\right)$ such that, for $d /(4 r)>\alpha+d / 4-1 / 2$,

$$
\left\|J_{\alpha}(v)\left(t_{0}, t, \cdot\right)\right\|_{q} \leqslant C \int_{t_{0}}^{t}(t-s)^{-\alpha-(d+2) / 4+d /(4 r)}\|v(s, \cdot)\|_{\rho} \mathrm{d} s .
$$

We also need upper estimates of increments of the Green kernel $G$.

Lemma 1.8. For $\gamma<4-d$ and $\gamma \leqslant 2, \gamma^{\prime}<1-d / 4$, there exists $C>0$ such that, for $t>s$, $x, z \in D$,

$$
\begin{array}{r}
\int_{0}^{t} \int_{D}|G(t-u, x, y)-G(t-u, z, y)|^{2} \mathrm{~d} y \mathrm{~d} u \leqslant C|x-z|^{\gamma}, \\
\int_{0}^{s} \int_{D}|G(t-u, x, y)-G(s-u, x, y)|^{2} \mathrm{~d} y \mathrm{~d} u \leqslant C|t-s|^{\gamma^{\prime}}, \\
\int_{s}^{t} \int_{D}|G(t-u, x, y)|^{2} \mathrm{~d} y \mathrm{~d} u \leqslant C|t-s|^{\gamma^{\prime}} .
\end{array}
$$

Proof. To prove these inequalities, we use the series decomposition (1.5) of $G$. Since the family $\left(\varepsilon_{k}\right)_{k \in \mathbb{N}}$ is an orthonormal basis of $L^{2}(D)$, we have 


$$
\begin{aligned}
& \int_{0}^{t} \int_{D}|G(t-u, x, y)-G(t-u, z, y)|^{2} \mathrm{~d} y \mathrm{~d} u \\
& \quad=\int_{0}^{t} \int_{D}\left|\sum_{k \in \mathbb{N}^{d}} \varepsilon_{k}(y) \exp \left(-\lambda_{k}^{2}(t-u)\right)\left[\varepsilon_{k}(z)-\varepsilon_{k}(x)\right]\right|^{2} \mathrm{~d} y \mathrm{~d} u \\
& \quad=\sum_{k \in \mathbb{N}^{d}} \int_{0}^{t} \exp \left(-2 \lambda_{k}^{2}(t-u)\right)\left|\varepsilon_{k}(z)-\varepsilon_{k}(x)\right|^{2} \mathrm{~d} u \\
& \quad=\sum_{k \in \mathbb{N}^{d, *}}\left|\varepsilon_{k}(z)-\varepsilon_{k}(x)\right|^{2}\left[1-\exp \left(-2 \lambda_{k}^{2} t\right)\right] \lambda_{k}^{-2} 2^{-1} .
\end{aligned}
$$

Since $k \neq 0$, we have

$$
\left|\varepsilon_{k}(z)-\varepsilon_{k}(x)\right| \leqslant C\left(\left[\lambda_{k}^{1 / 2}|z-x|\right] \wedge 1\right)
$$

and for $\alpha \in[0,1[$,

$$
\begin{aligned}
\int_{0}^{t} \int_{D}|G(t-u, x, y)-G(t-u, z, y)|^{2} \mathrm{~d} y \mathrm{~d} u & \leqslant C|z-x|^{2 \alpha} \sum_{k \in \mathbb{N} d, *} \lambda_{k}^{-2+\alpha}\left(1-\exp \left(-2 \lambda_{k}^{2} t\right)\right) \\
& \leqslant C|z-x|^{2 \alpha} \sum_{k \in \mathbb{N} d, *} \lambda_{k}^{-2+\alpha}
\end{aligned}
$$

Comparing the series $\sum_{k \in \mathbb{N} d, *} \lambda_{k}^{-u}$ with a multiple integral, we deduce that it converges if $u>d / 2$. So taking $0<\alpha<(4-d) / 2$ and $\alpha \leqslant 1$, we deduce (1.16).

We now prove inequality (1.17). Using (1.5), we obtain

$$
\begin{aligned}
& \int_{0}^{s} \int_{D}|G(t-u, x, y)-G(s-u, x, y)|^{2} \mathrm{~d} y \mathrm{~d} u \\
& \quad=\sum_{k \in \mathbb{N}^{d}}\left|\varepsilon_{k}(x)\right|^{2} \int_{0}^{s}\left[\exp \left(-\lambda_{k}^{2}(t-s)-1\right]^{2} \exp \left(-2 \lambda_{k}^{2}(s-u)\right) \mathrm{d} u\right. \\
& \quad \leqslant C \sum_{k \in \mathbb{N}^{d, *}} \lambda_{k}^{-2}\left[\exp \left(-\lambda_{k}^{2}(t-s)\right)-1\right]^{2}\left[1-\exp \left(-2 \lambda_{k}^{2} s\right)\right] .
\end{aligned}
$$

Since $\mid 1-\exp \left(-2 \lambda_{k}^{2} s \mid \leqslant 1 \wedge\left(2 \lambda_{k}^{2} s\right)\right.$, taking $\alpha \in[0,1]$, we have, for $s \leqslant T$,

$$
\int_{0}^{s} \int_{D}|G(t-u, x, y)-G(s-u, x, y)|^{2} \mathrm{~d} y \mathrm{~d} u \leqslant C \sum_{k \in \mathbb{N}^{d, *}}|t-s|^{\alpha} \lambda_{k}^{2 \alpha-2} .
$$

The series converges if and only if $\alpha<1-d / 4$, which yields (1.17).

The proof of (1.18), similar to the previous one, is omitted. 


\section{Existence and uniqueness of the solution to the Cahn- Hilliard stochastic equation}

Suppose that Assumptions 1-3 are satisfied; we first solve the problem when the function $f$ is truncated to have globally Lipschitz coefficients.

\subsection{The case of truncated $f$}

Let $n>0$ and denote by $K_{n}:\left[0,+\infty\left[\rightarrow\left[0,+\infty\left[\right.\right.\right.\right.$ a $\mathscr{C}^{1}$ function such that $\left|K_{n}\right| \leqslant 1$, $\left|K_{n}^{\prime}\right| \leqslant 2$ and

$$
K_{n}(x)= \begin{cases}1 & \text { if } x<n, \\ 0 & \text { if } x \geqslant n+1 .\end{cases}
$$

Let $\beta \in\left[q,+\infty\left[\right.\right.$ if $d \in\{1,2\}$; if $d=3$, choose $\beta$ such that $q \leqslant \beta<6 q /(6-q)^{+}$. We will prove the existence and uniqueness of the solution to the SPDE

$$
\begin{aligned}
u_{n}(t, x)= & \int_{D} G(t, x, y) u_{0}(y) \mathrm{d} y+\int_{0}^{t} \int_{D} \Delta G(t-s, x, y) K_{n}\left(\left\|u_{n}(s, \cdot)\right\|_{q}\right) f\left(u_{n}(s, y)\right) \mathrm{d} y \mathrm{~d} s \\
& +\int_{0}^{t} \int_{D} G(t-s, x, y) \sigma\left(u_{n}(s, y)\right) W(\mathrm{~d} y, \mathrm{~d} s)
\end{aligned}
$$

in the set $\mathscr{H}$ of $L^{q}(D)$-valued $\mathscr{F}_{t}$-adapted random processes $u(t, \cdot)$ such that the norm

$$
\|u\|_{\mathscr{H}}=\sup _{0 \leqslant t \leqslant T} \mathrm{E}\left(\|u(t, \cdot)\|_{q}^{\beta}\right)^{1 / \beta}
$$

is finite. Define two nonlinear operators on $\mathscr{H}$ by

$$
\begin{aligned}
H_{n}: \mathscr{H} & \rightarrow \mathscr{H} \\
u & \mapsto \int_{0}^{t} \int_{D} \Delta G(t-s, x, y) K_{n}\left(\|u(s, \cdot)\|_{q}\right) f(u(s, y)) \mathrm{d} y \mathrm{~d} s
\end{aligned}
$$

and

$$
\begin{aligned}
L: \mathscr{H} & \rightarrow \mathscr{H} \\
u & \mapsto \int_{0}^{t} \int_{D} G(t-s, x, y) \sigma(u(s, y)) W(\mathrm{~d} y, \mathrm{~d} s) .
\end{aligned}
$$

We prove that for $T$ small enough, $H_{n}$ and $L$ are contractions.

We begin by studying $H_{n}$. Let $u \in \mathscr{H}$; (1.12) applied with $1 \leqslant \rho \leqslant q, 1 \leqslant r_{1}<\infty$ if $d=2$ and $r_{1}<3$ if $d=3$ such that $1 / q=1 / r_{1}+1 / \rho-1$, implies that

$$
\left\|H_{n}(u)(t, \cdot)\right\|_{q} \leqslant \int_{0}^{t}|t-s|^{-(d+2) / 4+d /\left(4 r_{1}\right)}\left\|f(u(s, \cdot)) K_{n}\left(\|u(s, \cdot)\|_{q}\right)\right\|_{\rho} \mathrm{d} s .
$$


Taking $3 \rho=q$, we have $1 / r_{1}=1-2 / q$, which satisfies $r_{1}<+\infty$ if $d=2$ and $r_{1}<3$ if $q=3$. Also

$$
\left\|H_{n}(u)(t, \cdot)\right\|_{q} \leqslant C(n+1)^{3} T^{1+d /\left(4 r_{1}\right)-(d+2) / 4},
$$

so that

$$
\left\|H_{n}(u)\right\|_{\mathscr{B}}<\infty
$$

hence $H_{n}$ is an operator mapping $\mathscr{H}$ into itself.

Let $u$ and $v$ belong to $\mathscr{H}$; notice that

$$
\left\|K_{n}\left(\|u(s, \cdot)\|_{q}\right) f(u(s, \cdot))-K_{n}\left(\|v(s, \cdot)\|_{q}\right) f(v(s, \cdot))\right\|_{\rho} \leqslant C_{n}\|u(s, \cdot)-v(s, \cdot)\|_{q} \cdot
$$

Indeed, without any loss of generality, we suppose that $\|u(s, \cdot)\|_{q} \leqslant\|v(s, \cdot)\|_{q}$; then

$$
\begin{aligned}
&\left\|K_{n}\left(\|u(s, \cdot)\|_{q}\right) f(u(s, \cdot))-K_{n}\left(\|v(s, \cdot)\|_{q}\right) f(v(s, \cdot))\right\|_{\rho} \\
& \leqslant K_{n}\left(\|v(s, \cdot)\|_{q}\right)\|f(v(s, \cdot))-f(u(s, \cdot))\|_{\rho} \\
& \quad+\left\|\left[K_{n}\left(\|u(s, \cdot)\|_{q}\right)-K_{n}\left(\|v(s, \cdot)\|_{q}\right)\right] f(u(s, \cdot))\right\|_{\rho} .
\end{aligned}
$$

Assumption 1 implies that

$$
\begin{aligned}
& K_{n}\left(\|v(s, \cdot)\|_{q}\right)\|f(v(s, \cdot))-f(u(s, \cdot))\|_{\rho} \\
& \quad \leqslant C\left[\|u(s, \cdot)-v(s, \cdot)\|_{q}\left(1+\|v(s, \cdot)\|_{q}^{2}+\|u(s, \cdot)\|_{q}^{2}\right)\right] \times K_{n}\left(\|v(s, \cdot)\|_{q}\right) \\
& \quad \leqslant C\|u(s, \cdot)-v(s, \cdot)\|_{q} \times\left[1+2(n+1)^{2}\right] .
\end{aligned}
$$

For the second term, notice that if $\|v(s, \cdot)\|_{q} \geqslant\|u(s, \cdot)\|_{q}>n+1$, then

$$
\left\|\left[K_{n}\left(\|u(s, \cdot)\|_{q}\right)-K_{n}\left(\|v(s, \cdot)\|_{q}\right)\right] f(u(s, \cdot))\right\|_{\rho}=0 ;
$$

this yields

$$
\begin{aligned}
& \left\|\left[K_{n}\left(\|u(s, \cdot)\|_{q}\right)-K_{n}\left(\|v(s, \cdot)\|_{q}\right)\right] f(u(s, \cdot))\right\|_{\rho} \leqslant C\left(1+(n+1)^{3}\right)\left|\|u(s, \cdot)\|_{q}-\|v(s, \cdot)\|_{q}\right| \\
& \quad \leqslant C\left(1+(n+1)^{3}\right)\|u(s, \cdot)-v(s, \cdot)\|_{q} .
\end{aligned}
$$

Inequalities (2.8) and (2.9) imply (2.7). Using (2.7), (1.12) and Hölder's inequality with respect to $(t-s)^{-(d+2) / 4+d /\left(4 r_{1}\right)} \mathrm{d} s$, we conclude that

$$
\begin{aligned}
\left\|H_{n}(u)(t, \cdot)-H_{n}(v)(t, \cdot)\right\|_{\mathscr{H}}^{\beta} \leqslant & C_{n} \sup _{t \in[0, T]}\left[\left(\int_{0}^{t}(t-s)^{-(d+2) / 4+d /\left(4 r_{1}\right)} \mathrm{d} s\right)^{\beta-1}\right. \\
& \left.\times \int_{0}^{t}(t-s)^{-(d+2) / 4+d /\left(4 r_{1}\right)} \mathrm{E}\left(\|u(s, \cdot)-v(s, \cdot)\|_{q}^{\beta}\right) \mathrm{d} s\right] \\
\leqslant & C_{n} T^{\beta\left([2-d] / 4+d /\left(4 r_{1}\right)\right)}\|u-v\|_{\mathscr{H}}^{\beta} .
\end{aligned}
$$

If $T$ satisfies $C_{n} T^{\left(d /\left(4 r_{1}\right)+[2-d] / 4\right) \beta}<1$, the map $H_{n}$ is a contraction on $\mathscr{H}$. 
We now turn to $L$. Let $u$ belong to $\mathscr{H}$; Burkholder's inequality, (1.6) and Assumption 2 imply that, for an absolute constant $C$ depending on $G$,

$$
\begin{aligned}
& \sup _{(t, x) \in[0, T] \times D} \mathrm{E}\left(\left|\int_{0}^{t} \int_{D} G(t-s, x, y) \sigma(u(s, y)) W(\mathrm{~d} y, \mathrm{~d} s)\right|^{\beta}\right) \\
& \leqslant \sup _{(t, x) \in[0, T] \times D} \mathrm{E}\left(\left|\int_{0}^{t} \int_{D} G^{2}(t-s, x, y) \sigma^{2}(u(s, y)) \mathrm{d} y \mathrm{~d} s\right|^{\beta / 2}\right) \\
& \leqslant C\|\sigma\|_{\infty}^{2}<+\infty
\end{aligned}
$$

this implies $\|L(u)\|_{\mathscr{H}}<\infty$, for every $u \in \mathscr{H}$. Let $u, v \in \mathscr{H}$; then, since $\beta \geqslant q$, we have

$$
\mathrm{E}\left(\|L(u)(s, \cdot)-L(v)(s, \cdot)\|_{q}^{\beta}\right) \leqslant C \int_{D} \mathrm{E}\left(|L(u)(s, x)-L(v)(s, x)|^{\beta}\right) \mathrm{d} x .
$$

Burkholder's inequality and (1.13) applied with $\beta / 2$ instead of $q$ and $q / 2$ instead of $\rho$, $1 / r_{2}=1+2 / \beta-2 / q>0$ (indeed, if $d=3$, the condition $\beta<6 q /(6-q)^{+}$yields $r_{2} \leqslant \frac{3}{2}$ ), imply, for $t \leqslant T$,

$$
\begin{aligned}
& \mathrm{E}\left(\|L(u)(t, \cdot)-L(v)(t, \cdot)\|_{q}^{\beta}\right) \\
& \quad \leqslant \int_{D} \mathrm{E}\left(\left|\int_{0}^{t} \int_{D} G^{2}(t-s, x, y)\right| \sigma(u(s, y))-\left.\left.\sigma(v(s, y))\right|^{2} \mathrm{~d} y \mathrm{~d} s\right|^{\beta / 2}\right) \mathrm{d} x \\
& \quad \leqslant C \mathrm{E}\left(\left|\int_{0}^{t}(t-s)^{-d / 2+d /\left(4 r_{2}\right)}\|u(s, \cdot)-v(s, \cdot)\|_{q}^{2} \mathrm{~d} s\right|^{\beta / 2}\right) \\
& \quad \leqslant C T^{\beta\left(1-d / 2+d /\left(4 r_{2}\right)\right)} \sup _{s \in[0, T]} \mathrm{E}\left(\|u(s, \cdot)-v(s, \cdot)\|_{q}^{\beta}\right) .
\end{aligned}
$$

Again, for $T$ small enough, $L$ is a contraction on $\mathscr{H}$.

In conclusion, the operator $H_{n}+L$ working on $\mathscr{H}$ is a contraction for $T \leqslant T_{0}$. Hence, it admits a unique fixed point in the set

$$
\left\{u \in \mathscr{H} \text { such that } u(0, \cdot)=u_{0}\right\} .
$$

By a classical argument, because $T_{0}$ does not depend on $u_{0}$, we can construct by concatenation on every interval $[0, T]$ a unique solution $u_{n}$ to the SPDE (2.2).

\subsection{Existence and uniqueness of the solution to (1.10)}

Again let $\beta \in\left[q,+\infty\left[\right.\right.$ if $d=1,2$, and $\beta \in\left[q, 6 q /(6-q)^{+}[\right.$if $d=3$; set

$$
\tau_{n}=\inf \left\{t \geqslant 0 \mid\left\|u_{n}(t, \cdot)\right\|_{q} \geqslant n\right\} .
$$


By uniqueness of the solution to (2.2), the local property of stochastic integrals yields, for $m>n, u_{m}(t, \cdot)=u_{n}(t, \cdot)$ if $t \leqslant \tau_{n}$, so that we can define a process $u$ by $u(t, \cdot)=u_{n}(t, \cdot)$ on $t \leqslant \tau_{n}$. Set $\tau_{\infty}=\lim _{n} \tau_{n}$; clearly $u$ is a solution to the SPDE (1.10) on the interval [0, $\tau_{\infty}$ ), and is unique. We just need to prove that $\tau_{\infty}=+\infty$ a.s., and use an argument similar to that of Da Prato and Debussche (1996, Section 2.1).

Let $L$ be defined by (2.5) and set $v_{n}=u_{n}-L\left(u_{n}\right)$; then, for every $T>0, v_{n}$ is the weak solution on $[0, T]$ to the SPDE

$$
\begin{aligned}
& \frac{\partial v_{n}}{\partial t}(t, x)+\Delta^{2} v_{n}(t, x)-\Delta\left[K_{n}\left(\| v_{n}(t, \cdot)+L\left(u_{n}(t, \cdot) \|_{q}\right) f\left(v_{n}(t, x)+L\left(u_{n}\right)(t, x)\right)\right]=0,\right. \\
& v_{n}(0, \cdot)=u_{0}(\cdot), \\
& \frac{\partial v_{n}}{\partial n}=\frac{\partial \Delta v_{n}}{\partial n}=0 \text { on } \partial D .
\end{aligned}
$$

Again (2.13) is formal and can be made rigorous as in (1.9) by requiring that, for any $\phi \in C^{4}(D)$ such that $\phi$ satisfies (1.2),

$$
\begin{aligned}
\int_{D}\left[v_{n}(t, x)-u_{0}(t, x)\right] \phi(x) \mathrm{d} x= & -\int_{0}^{t} \int_{D} \Delta^{2} \phi(x) v_{n}(s, x) \mathrm{d} x \mathrm{~d} s \\
& +\int_{0}^{t} \int_{D} \Delta \phi(x) K_{n}\left(\left\|v_{n}(s, \cdot)+L\left(u_{n}\right)(s, \cdot)\right\|_{q}\right) f\left(v_{n}(s, x)\right. \\
& +L\left(u_{n}(s, x)\right) \mathrm{d} x \mathrm{~d} s .
\end{aligned}
$$

Since $\sigma$ is bounded, (2.11) yields, for any $p, \delta \in] 1,+\infty[$,

$$
\sup _{n} \sup _{t \in[0, T]} \sup _{x \in D} \mathrm{E}\left(\left|L\left(u_{n}\right)(t, x)\right|^{2 p \delta}\right)<+\infty .
$$

Lemma 1.8 and Burkholder's inequality imply that, for every $\gamma<(4-d), \quad \gamma \leqslant 2$, $\gamma^{\prime}<1-d / 4$, and $\alpha>1, T>0,\left(t, t^{\prime}, x, x^{\prime}\right) \in[0, T]^{2} \times D^{2}$, for all $n \in \mathbb{N}$ :

$$
\mathrm{E}\left(\left|L\left(u_{n}\right)(t, x)-L\left(u_{n}\right)\left(t^{\prime}, x^{\prime}\right)\right|^{2 \alpha}\right) \leqslant C\left(\left|t-t^{\prime}\right|^{\gamma \prime}+\left|x-x^{\prime}\right|^{\gamma}\right)^{\alpha} .
$$

Inequalities (2.14) and (2.15) and the Garsia-Rodemich-Ramsay lemma (see Garsia 1972) yield, if $\left\|L\left(u_{n}\right)\right\|_{\infty}=\sup _{t \in[0, T]} \sup _{x \in D}\left|L\left(u_{n}\right)(t, x)\right|$,

$$
\sup _{n} \mathrm{E}\left(\left\|L\left(u_{n}\right)\right\|_{\infty}^{2 p \delta}\right)<\infty .
$$

On the other hand, since $G(t, x, y)=G(t, y, x)$ and $\int_{D}|G(t, x, y)| \mathrm{d} y<+\infty$, for every $q \in[1,+\infty[$,

$$
\sup _{t \in[0, T]}\left\|G_{t} u_{0}\right\|_{q} \leqslant C\left\|u_{0}\right\|_{q} .
$$

We just need to prove a uniform upper estimate for $H_{n}\left(u_{n}\right)$. Since the functional $H_{n}$ has a regularizing effect, we first show that $u_{n}$ belongs to the sets $L^{a}\left([0, T] ; L^{q}(D)\right)$ for $2 \leqslant$ $a<+\infty$. As in Da Prato and Debussche (1996), we shall prove a priori estimates on $v_{n}$. 
Recall that for $A=-\Delta, \alpha \neq 0$ and $u \in \operatorname{Dom}\left(A^{\alpha}\right)$,

$$
A^{\alpha} u=\sum_{k \in \mathbb{N}^{d, *}} \lambda_{k}^{\alpha}\left\langle\varepsilon_{k}, u\right\rangle \varepsilon_{k}
$$

$A^{\alpha} u$ exists for every $u$ such that $\sum_{k \in \mathbb{N} d, *} \lambda_{k}^{2 \alpha}\left\langle e_{k}, u\right\rangle^{2}<\infty$. In what follows, for a function $u:[0, T] \times D \rightarrow \mathbb{R}$, we will set

$$
m(u)(t)=\left\langle\varepsilon_{0}, u(t, \cdot)\right\rangle=\pi^{-d / 2} \int_{D} u(t, x) \mathrm{d} x \quad \text { and } \quad \tilde{u}(t, y)=u(t, y)-m(u)(t) .
$$

Notice that $A^{\alpha} \tilde{u}=A^{\alpha} u$ for $\alpha \neq 0, u \in \operatorname{Dom}\left(A^{\alpha}\right)$. Apply $A^{-1}$ to equation (2.24) and take its scalar product in $L^{2}(D)$ with $\tilde{v}_{n}(t, \cdot)$; this leads to

$$
\begin{aligned}
& \left\|A^{-1 / 2} \tilde{\boldsymbol{v}}_{n}(t, \cdot)\right\|_{2}^{2}-\left\|A^{-1 / 2} \tilde{\boldsymbol{v}}_{n}(0, \cdot)\right\|_{2}^{2}+\int_{0}^{t}\left\|A^{1 / 2} \boldsymbol{v}_{n}(s, \cdot)\right\|_{2}^{2} \mathrm{~d} s \\
& \quad+\int_{0}^{t} K_{n}\left(\left\|\boldsymbol{v}_{n}(s, \cdot)+L\left(u_{n}\right)(s, \cdot)\right\|_{q}\right) \int_{D} f\left(v_{n}(s, x)+L\left(u_{n}\right)(s, x)\right) \tilde{v}_{n}(s, x) \mathrm{d} x \mathrm{~d} s=0 .
\end{aligned}
$$

This equation is justified because $v_{n}$ belongs to $L^{\infty}\left([0, T], L^{q}(D)\right)$; since for $q \geqslant 2$, $L^{q}(D) \subset \operatorname{Dom}\left(A^{-1 / 2}\right)$ and the first two terms are well defined so that $\int_{0}^{t}\left\|A^{1 / 2} v_{n}(s, \cdot)\right\|_{2}^{2} \mathrm{~d} s$ converges too and $v_{n}(s, \cdot)$ belongs to $\operatorname{Dom}\left(A^{-1 / 2}\right)$.

Let us deal with the last term of (2.18):

$$
\begin{aligned}
\int_{D} f\left(v_{n}(t, x)+L\left(u_{n}\right)(t, x)\right) \tilde{v}_{n}(t, x) \mathrm{d} x \\
=\int_{D} f\left(v_{n}(t, x)+L\left(u_{n}\right)(t, x)\right)\left[v_{n}(t, x)+L\left(u_{n}\right)(t, x)\right] \mathrm{d} x \\
\quad-\int_{D} f\left(v_{n}(t, x)+L\left(u_{n}\right)(t, x)\right)\left[m\left(v_{n}\right)(t)+L\left(u_{n}\right)(t, x)\right] \mathrm{d} x .
\end{aligned}
$$

The polynomial $f$ is of degree 3 with positive dominant coefficient; hence, $\lim _{|x| \rightarrow+\infty} x f(x)=$ $+\infty$, and there exist $a, C$ and $c>0$ such that

$$
x f(x) \geqslant \frac{7}{8} a x^{4}-c, \quad|f(x)| \leqslant \frac{5}{4} a|x|^{3}+C .
$$

The lower estimate of $x f(x)$ implies

$$
\int_{D} f\left(v_{n}(t, x)+L\left(u_{n}(t, x)\right)\right)\left(v_{n}(t, x)+L\left(u_{n}\right)(t, x)\right) \mathrm{d} x \geqslant \frac{7}{8} a\left\|v_{n}(t, \cdot)+L\left(u_{n}\right)(t, \cdot)\right\|_{4}^{4}-c .
$$

The upper estimate of $|f|$ and Hölder's inequality with conjugate exponents 4 and $\frac{4}{3}$ yield: 


$$
\begin{aligned}
& \int_{D} f\left(v_{n}(t, x)+L\left(u_{n}(t, x)\right)\right)\left(m\left(v_{n}\right)(t)+L\left(u_{n}\right)(t, x)\right) \mathrm{d} x \\
& \leqslant \leq \frac{5}{4} a \int_{D}\left|v_{n}(t, x)+L\left(u_{n}\right)(t, x)\right|^{3}\left(\left|m\left(v_{n}\right)(t)\right|+\left|L\left(u_{n}\right)(t, \cdot)\right|\right) \mathrm{d} x \\
& \quad+C \int_{D}\left(\left|m\left(v_{n}\right)(t)\right|+\left|L\left(u_{n}\right)(t, \cdot)\right|\right) \mathrm{d} x \\
& \leqslant \frac{5}{4} a\left[\left\|v_{n}(t, \cdot)+L\left(u_{n}\right)(t, \cdot)\right\|_{4}^{3}\left|m\left(v_{n}\right)(t)\right|+\left\|v_{n}(t, \cdot)+L\left(u_{n}\right)(t, \cdot)\right\|_{4}^{3}\left\|L\left(u_{n}\right)(t, \cdot)\right\|_{4}\right] \\
& \quad+C\left(\left|m\left(v_{n}\right)(t)\right|+\left\|L\left(u_{n}\right)(t, \cdot)\right\|_{4}\right) .
\end{aligned}
$$

Notice that there exists $c>0$ such that, for every $x$ and $y,|x|^{3}|y| \leqslant \frac{1}{4}|x|^{4}+c|y|^{4}$; this implies

$$
\begin{aligned}
& \int_{D} f\left(v_{n}(t, x)+L\left(u_{n}(t, x)\right)\right)\left(m\left(v_{n}\right)(t)+L\left(u_{n}\right)(t, x)\right) \mathrm{d} x \\
& \quad \leqslant \frac{5 a}{8}\left\|v_{n}(t, \cdot)+L\left(u_{n}\right)(t, \cdot)\right\|_{4}^{4}+C\left[m\left(v_{n}(t)\right)^{4}+\left\|L\left(u_{n}\right)(t, \cdot)\right\|_{4}^{4}\right] .
\end{aligned}
$$

$K_{n}$ is a positive bounded function; using (2.19)-(2.21), (2.18) yields

$$
\begin{aligned}
& \left\|A^{-1 / 2} \tilde{\boldsymbol{v}}_{n}(t, \cdot)\right\|_{2}^{2}-\left\|A^{-1 / 2} \tilde{\boldsymbol{v}}_{n}(0, \cdot)\right\|_{2}^{2}+\int_{0}^{t}\left\|A^{1 / 2} \boldsymbol{v}_{n}(s, \cdot)\right\|_{2}^{2} \mathrm{~d} s \\
& +\frac{a}{4} \int_{0}^{t} K_{n}\left(\left\|v_{n}(s, \cdot)+L\left(u_{n}\right)(s, \cdot)\right\|_{q}\right)\left\|v_{n}(s, \cdot)+L\left(u_{n}\right)(s, \cdot)\right\|_{4}^{4} \mathrm{~d} s \\
& \quad \leqslant \int_{0}^{t} C\left(1+m\left(v_{n}\right)(s)^{4}+\left\|L\left(v_{n}\right)(s, \cdot)\right\|_{4}^{4}\right) \mathrm{d} s .
\end{aligned}
$$

Taking the scalar product of the solution to (2.24) with the function $\varepsilon_{0}$, we obtain

$$
\begin{aligned}
\frac{\partial}{\partial t}\left\langle v_{n}(t, \cdot), \varepsilon_{0}\right\rangle & =0, \\
\left\langle v_{n}(0, \cdot), \varepsilon_{0}\right\rangle & =\left\langle u_{0}(\cdot), \varepsilon_{0}\right\rangle ;
\end{aligned}
$$

hence for $m\left(u_{0}\right)=\left\langle\varepsilon_{0}, u_{0}\right\rangle, m\left(v_{n}\right)(t)=m\left(u_{0}\right)$. Since $A^{-1 / 2} \tilde{u}_{0}=A^{-1 / 2} u_{0}$, equation (2.22) yields that, for every $T>0$,

$$
\begin{aligned}
& \left\|A^{-1 / 2} \tilde{\boldsymbol{v}}_{n}(T, \cdot)\right\|_{2}^{2}+\int_{0}^{T}\left\|A^{1 / 2} v_{n}(t, \cdot)\right\|_{2}^{2} \mathrm{~d} t \\
& +\int_{0}^{T} \frac{a}{4} K_{n}\left(\left\|v_{n}(t, \cdot)+L\left(u_{n}\right)(t, \cdot)\right\|_{q}\right)\left\|v_{n}(t, \cdot)+L\left(u_{n}\right)(t, \cdot)\right\|_{4}^{4} \mathrm{~d} t \\
& \quad \leqslant C \int_{0}^{T}\left(1+m\left(u_{0}\right)^{4}+\left\|L\left(u_{n}\right)(t, \cdot)\right\|_{4}^{4}\right) \mathrm{d} t+\left\|A^{-1 / 2} u_{0}(\cdot)\right\|_{2}^{2}
\end{aligned}
$$


This yields

$$
\begin{aligned}
& \int_{0}^{T} K_{n}\left(\left\|v_{n}(t, \cdot)+L\left(u_{n}\right)(t, \cdot)\right\|_{q}\right)\left\|v_{n}(t, \cdot)+L\left(u_{n}\right)(t, \cdot)\right\|_{4}^{4} \mathrm{~d} t \\
& \quad \leqslant C_{T}\left(1+m\left(u_{0}\right)^{4}+\left\|L\left(u_{n}\right)\right\|_{\infty}^{4}\right)+\left\|A^{-1 / 2} u_{0}(\cdot)\right\|_{2}^{2} .
\end{aligned}
$$

We need another estimate, for this we denote by $v_{n}^{m}$ the Galerkin approximation of $v_{n}$. We define $P_{m}$ to be the orthogonal projector on $\operatorname{Span}\left\{\varepsilon_{0}, \ldots, \varepsilon_{m}\right\}$. For every $\omega, v_{n}^{m}$ is the 'strong' solution of the following PDE:

$$
\begin{aligned}
& \frac{\partial v_{n}^{m}}{\partial t}(t, x)+\Delta^{2} v_{n}^{m}(t, x) \\
& \quad-\Delta\left[K_{n}\left(\left\|v_{n}^{m}(t, \cdot)+L\left(u_{n}\right)(t, \cdot)\right\|_{q}\right) P_{m}\left(f\left(v_{n}^{m}(t, x)+L\left(u_{n}\right)(t, x)\right)\right)\right]=0, \\
& v_{n}^{m}(0, \cdot)=P_{m}\left(u_{0}(\cdot)\right), \\
& \frac{\partial v_{n}^{m}}{\partial n}=\frac{\partial \Delta v_{n}^{m}}{\partial n}=0 \text { on } \partial D .
\end{aligned}
$$

The proof of existence and uniqueness of the processes $v_{n}^{m}$ is classical; we use deterministic methods and prove that $v_{n}^{m}$ is unique on some time interval $\left[0, t_{n}^{m}[\right.$. The following a priori estimates will prove that $t_{n}^{m}=+\infty$.

The boundary conditions satisfied by $v_{n}^{m}$ and the Green formula yield

$$
\int_{D} \Delta^{2} v_{n}^{m}(t, x) \times v_{n}^{m}(t, x) \mathrm{d} x=\left\|\Delta v_{n}^{m}(t, x)\right\|_{2}^{2} .
$$

We now take the scalar product in $L^{2}(D)$ of (2.24) with $v_{n}$; using the Green formula once more, we obtain

$$
\begin{aligned}
& \frac{1}{2} \frac{\partial}{\partial t}\left\|v_{n}^{m}(t, \cdot)\right\|_{2}^{2}+\int_{D} \Delta^{2} v_{n}^{m}(t, x) \times v_{n}^{m}(t, x) \mathrm{d} x \\
& \quad-K_{n}\left(\left\|v_{n}^{m}(t, \cdot)+L\left(u_{n}\right)(t, \cdot)\right\|_{q}\right) \int_{D} f\left(v_{n}^{m}(t, x)+L\left(u_{n}\right)(t, x)\right) \Delta v_{n}^{m}(t, x) \mathrm{d} x=0 .
\end{aligned}
$$

Thus

$$
\begin{aligned}
& \frac{1}{2} \frac{\partial}{\partial t}\left\|v_{n}^{m}(t, \cdot)\right\|_{2}^{2}+\left\|\Delta v_{n}^{m}(t, \cdot)\right\|_{2}^{2} \\
& =K_{n}\left(\left\|v_{n}^{m}(t, \cdot)+L\left(u_{n}\right)(t, \cdot)\right\|_{q}\right) \\
& \quad \times\left[\int_{D}\left[f\left(v_{n}^{m}(t, x)+L\left(u_{n}\right)(t, x)\right)-f\left(v_{n}^{m}(t, x)\right)\right] \Delta\left(v_{n}^{m}(t, x)\right) \mathrm{d} x\right. \\
& \left.\quad+\int_{D} f\left(v_{n}^{m}(t, x)\right) \Delta v_{n}^{m}(t, x) \mathrm{d} x\right] .
\end{aligned}
$$

$f$ is a polynomial of degree 3 ; thus, for every $x, y \in \mathbb{R}$, 


$$
|f(x+y)-f(x)| \leqslant C|y|\left[1+x^{2}+y^{2}\right] .
$$

Let $\epsilon>0$; Schwarz's inequality, (2.26) and the inequality $x y \leqslant x^{2} / \epsilon+\epsilon y^{2}$ imply

$$
\begin{aligned}
& \left|\int_{D}\left[f\left(v_{n}^{m}(t, x)+L\left(u_{n}\right)(t, x)\right)-f\left(v_{n}^{m}(t, x)\right)\right] \Delta v_{n}^{m}(t, x) \mathrm{d} x\right| \\
& \quad \leqslant \int_{D} C\left|L\left(u_{n}\right)(t, x)\right|\left[1+\left|L\left(u_{n}\right)(t, x)\right|^{2}+\left|v_{n}^{m}(t, x)\right|^{2}\right]\left|\Delta v_{n}^{m}(t, x)\right| \mathrm{d} x \\
& \quad \leqslant C\left\|L\left(u_{n}\right)\right\|_{\infty}\left[1+\left\|L\left(u_{n}\right)\right\|_{\infty}^{2}+\left\|v_{n}^{m}(t, \cdot)^{2}\right\|_{2}\right]\left\|\Delta v_{n}^{m}(t, \cdot)\right\|_{2} \\
& \quad \leqslant C \epsilon\left\|\Delta v_{n}^{m}(t, \cdot)\right\|_{2}^{2}+\frac{C}{\epsilon}\left[1+\left\|L\left(u_{n}\right)\right\|_{\infty}^{4}+\left\|v_{n}^{m}(t, \cdot)\right\|_{4}^{4}\right]\left\|L\left(u_{n}\right)\right\|_{\infty}^{2} .
\end{aligned}
$$

Let us now turn to the second term of the right-hand side of (2.25). First, let us prove that $\int_{D}\left(v_{n}^{m}(t, x)^{3}\right) \Delta v_{n}^{m}(t, x) \mathrm{d} x$ is negative. Integrating by parts and using the boundary conditions on $v_{n}^{m}$, we find if $d=2$ (the other cases are similar),

$$
\begin{aligned}
& \int_{0}^{\pi} \int_{0}^{\pi} \frac{\partial^{2}}{\partial x_{1}^{2}}\left(v_{n}^{m}\left(t, x_{1}, x_{2}\right)\right) \times v_{n}^{m}\left(t, x_{1}, x_{2}\right)^{3} \mathrm{~d} x_{1} \mathrm{~d} x_{2} \\
& =\int_{0}^{\pi}\left[\frac{\partial}{\partial x_{1}}\left(v_{n}^{m}\left(t, x_{1}, x_{2}\right)\right) \times v_{n}^{m}\left(t, x_{1}, x_{2}\right)^{3}\right]_{x_{1}=0}^{x_{1}=\pi} \mathrm{d} x_{2} \\
& \quad-3 \int_{0}^{\pi} \int_{0}^{\pi}\left[\frac{\partial}{\partial x_{1}}\left(v_{n}^{m}\left(t, x_{1}, x_{2}\right)\right)\right]^{2} v_{n}^{m}\left(t, x_{1}, x_{2}\right)^{2} \mathrm{~d} x_{1} \mathrm{~d} x_{2} \\
& =-3 \int_{0}^{\pi} \int_{0}^{\pi}\left[\frac{\partial}{\partial x_{1}}\left(v_{n}^{m}\left(t, x_{1}, x_{2}\right)\right)\right]^{2} v_{n}^{m}\left(t, x_{1}, x_{2}\right)^{2} \mathrm{~d} x_{1} \mathrm{~d} x_{2} \leqslant 0 ;
\end{aligned}
$$

this yields $\int_{D} v_{n}^{m}(t, x)^{3} \Delta v_{n}^{m}(t, x) \mathrm{d} x \leqslant 0$. Since the main coefficient of $f$ is positive, this implies

$$
\begin{aligned}
\int_{D} f\left(v_{n}^{m}(t, x)\right) \Delta v_{n}^{m}(t, x) \mathrm{d} x & \leqslant C \int_{D}\left(1+\left|v_{n}^{m}(t, x)\right|^{2}\right)\left|\Delta v_{n}^{m}(t, x)\right| \mathrm{d} x \\
& \leqslant C \epsilon\left\|\Delta v_{n}^{m}(t, \cdot)\right\|_{2}^{2}+\frac{C}{\epsilon}\left[1+\left\|v_{n}^{m}(t, \cdot)\right\|_{4}^{4}\right] .
\end{aligned}
$$

Furthermore, $\left\|K_{n}\right\| \infty \leqslant 1$; using (2.27) and (2.28), equation (2.25) becomes

$$
\begin{aligned}
\frac{1}{2} \frac{\partial}{\partial t}\left\|v_{n}^{m}(t, \cdot)\right\|_{2}^{2}+\left\|\Delta v_{n}^{m}(t, \cdot)\right\|_{2}^{2} \leqslant & C_{1} \epsilon\left\|\Delta v_{n}^{m}(t, \cdot)\right\|_{2}^{2}+\frac{C_{2}}{\epsilon}\left[1+\left\|L\left(u_{n}\right)\right\|_{\infty}^{4}+\left\|v_{n}^{m}(t, \cdot)\right\|_{4}^{4}\right] \\
& \times\left(1+\left\|L\left(u_{n}\right)\right\|_{\infty}^{2}\right) K_{n}\left(\left\|v_{n}^{m}(t, \cdot)+L\left(u_{n}\right)(t, \cdot)\right\|_{q}\right) .
\end{aligned}
$$

Choose $\epsilon=1 /\left(2 C_{1}\right)$; then 


$$
\begin{aligned}
& \frac{1}{2} \frac{\partial}{\partial t}\left\|v_{n}^{m}(t, \cdot)\right\|_{2}^{2}+\frac{1}{2}\left\|\Delta v_{n}^{m}(t, \cdot)\right\|_{2}^{2} \\
& \quad \leqslant 2 C_{1} C_{2}\left[1+\left\|L\left(u_{n}\right)\right\|_{\infty}^{4}+\left\|v_{n}^{m}(t, \cdot)\right\|_{4}^{4}\right]\left(1+\left\|L\left(u_{n}\right)\right\|_{\infty}^{2}\right) K_{n}\left(\left\|v_{n}^{m}(t, \cdot)+L\left(u_{n}\right)(t, \cdot)\right\|_{q}\right) .
\end{aligned}
$$

Integrating on $[0, t]$, we obtain

$$
\begin{aligned}
\left\|v_{n}^{m}(t, \cdot)\right\|_{2}^{2} & +\int_{0}^{t}\left\|\Delta v_{n}^{m}(s, \cdot)\right\|_{2}^{2} \mathrm{~d} s \leqslant\left\|P_{m} u_{0}\right\|_{2}^{2}+C T\left(1+\left\|L\left(u_{n}\right)\right\|_{\infty}^{6}\right) \\
& +C\left(1+\left\|L\left(u_{n}\right)\right\|_{\infty}^{2}\right) \int_{0}^{t}\left\|v_{n}^{m}(s, \cdot)\right\|_{4}^{4} K_{n}\left(\left\|v_{n}^{m}(s, \cdot)+L\left(u_{n}\right)(s, \cdot)\right\|_{q}\right) \mathrm{d} s .
\end{aligned}
$$

Therefore the equality $m\left(v_{n}^{m}(t, \cdot)\right)=m\left(v_{n}(t, \cdot)\right)=m\left(u_{0}(\cdot)\right)$ implies

$$
\begin{aligned}
& \left\|v_{n}^{m}(t, \cdot)\right\|_{2}^{2}+\int_{0}^{t}\left[\left\|\Delta v_{n}^{m}(s, \cdot)\right\|_{2}^{2}+m\left(v_{n}^{m}(s, \cdot)\right)^{2}\right] \mathrm{d} s \leqslant\left\|u_{0}\right\|_{2}^{2}+C T\left(1+\left\|L\left(u_{n}\right)\right\|_{\infty}^{6}+m\left(u_{0}\right)\right) \\
& \quad+C\left(1+\left\|L\left(u_{n}\right)\right\|_{\infty}^{2}\right) \int_{0}^{t}\left\|v_{n}^{m}(s, \cdot)\right\|_{4}^{4} K_{n}\left(\left\|v_{n}^{m}(s, \cdot)+L\left(u_{n}\right)(s, \cdot)\right\|_{q}\right) \mathrm{d} s .
\end{aligned}
$$

The norm $\left(\|\Delta \cdot\|_{L^{2}(D)}^{2}+m(\cdot)^{2}\right)^{1 / 2}$ is equivalent to the Sobolev norm of $W^{2,2}(D)$ (see, for example, Da Prato and Debussche 1996, p. 245). The sequence $\left(v_{n}^{m}\right)_{m \in \mathbb{N}}$ is bounded in $L^{2}\left([0, T], W^{2,2}(D)\right)$. Thus, $t_{n}^{m}=\infty$ and this sequence converges as $m \rightarrow+\infty$ in the weak* topology of $L^{2}\left([0, T], W^{2,2}(D)\right)$. Its weak limit is the weak solution to $(2.13)$ and hence is equal to $v_{n}$. Therefore, $v_{n}$ belongs to $L^{2}\left([0, T], W^{2,2}(D)\right)$, and we can repeat the preceding computation with $v_{n}$ instead of $v_{n}^{m}$, which yields

$$
\begin{aligned}
& \left\|v_{n}(t, \cdot)\right\|_{2}^{2}+\int_{0}^{t}\left[\left\|\Delta v_{n}(s, \cdot)\right\|_{2}^{2}+m\left(v_{n}(s, \cdot)\right)^{2}\right] \mathrm{d} s \leqslant\left\|u_{0}\right\|_{2}^{2}+C T\left(1+\left\|L\left(u_{n}\right)\right\|_{\infty}^{6}+m\left(u_{0}\right)\right) \\
& \quad+C\left(1+\left\|L\left(u_{n}\right)\right\|_{\infty}^{2}\right) \int_{0}^{t}\left\|v_{n}(s, \cdot)\right\|_{4}^{4} K_{n}\left(\left\|v_{n}(s, \cdot)+L\left(u_{n}\right)(s, \cdot)\right\|_{q}\right) \mathrm{d} s .
\end{aligned}
$$

Inequality (2.23) and Schwarz's inequality imply that

$$
\begin{aligned}
& \left\|v_{n}(t, \cdot)\right\|_{2}^{2}+\int_{0}^{t}\left[\left\|\Delta v_{n}(s, \cdot)\right\|_{2}^{2}+m\left(v_{n}(s, \cdot)\right)^{2}\right] \mathrm{d} s \\
& \quad \leqslant\left\|u_{0}\right\|_{2}^{2}+C_{T}\left(1+\left\|L\left(u_{n}\right)\right\|_{\infty}^{6}\right)+C_{T}\left(1+\left\|L\left(u_{n}\right)\right\|_{\infty}^{2}\right)\left[\left\|A^{-1 / 2} u_{0}\right\|_{2}^{2}+m\left(u_{0}\right)^{4}\right] .
\end{aligned}
$$

Inequality (2.16) yields that, for $\beta \in] 1,+\infty[$,

$$
\begin{array}{r}
\sup _{n} \mathrm{E}\left(\sup _{t \in[0, T]}\left\|v_{n}(t, \cdot)\right\|_{2}^{2 \beta}\right)<\infty, \\
\sup _{n} \mathrm{E}\left(\left[\int_{0}^{T}\left\{\left\|\Delta v_{n}(t, \cdot)\right\|_{2}^{2}+m\left(v_{n}(t, .)\right)^{2}\right\} \mathrm{d} t\right]^{\beta}\right)<\infty .
\end{array}
$$


Furthermore, by Sobolev's embedding theorem (Adams 1975, Corollary 5.16) there exists $C>0$ such that for $r \geqslant 2, d<4$, if $u \in W^{2,2}(D)$,

$$
\|u\|_{L^{r}(D)} \leqslant C\|u\|_{W^{2,2}(D)} .
$$

Thus, (2.30) becomes for $2 \leqslant r<+\infty$,

$$
\sup _{n} \mathrm{E}\left(\left[\int_{0}^{T}\left\|v_{n}(t, \cdot)\right\|_{r}^{2} \mathrm{~d} t\right]^{\beta}\right)<\infty .
$$

Inequalities (2.16), (2.29) and (2.31) imply for $2 \leqslant r<+\infty$,

$$
\begin{array}{r}
\sup _{n} \mathrm{E}\left(\sup _{t \in[0, T]}\left\|u_{n}(t, \cdot)\right\|_{2}^{2 \beta}\right)<\infty, \\
\sup _{n} \mathrm{E}\left(\left[\int_{0}^{T}\left\|u_{n}(t, \cdot)\right\|_{r}^{2} \mathrm{~d} t\right]^{\beta}\right)<\infty .
\end{array}
$$

Let us use the interpolation method to prove that $u_{n}$ belongs a.s. to $L^{a}\left([0, T], \mathrm{L}^{q}(D)\right)$, with $+\infty>r \geqslant q \geqslant 2, a \geqslant 1 \vee 2 q / r$. Hölder's inequality implies, if $q=(1-\lambda) 2+r \lambda$, for $\lambda \in[0,1]$, that

$$
\int_{0}^{T}\left\|u_{n}(t, \cdot)\right\|_{q}^{a} \mathrm{~d} t \leqslant \int_{0}^{T}\left\|u_{n}(t, \cdot)\right\|_{2}^{2 a(1-\lambda) / q}\left\|u_{n}(t, \cdot)\right\|_{r}^{a r \lambda / q} \mathrm{~d} t .
$$

Taking $\lambda=2 q /($ ar $)$, we obtain

$$
\int_{0}^{T}\left\|u_{n}(t, \cdot)\right\|_{q}^{a} \mathrm{~d} t \leqslant \sup _{t \in[0, T]}\left\|u_{n}(t, \cdot)\right\|_{2}^{[2 / q] a(1-\lambda)} \times \int_{0}^{T}\left\|u_{n}(t, \cdot)\right\|_{r}^{2} \mathrm{~d} t
$$

(2.32) and (2.33) imply that for $q \in[2, \infty[$ and $a \in[q,+\infty[$,

$$
\sup _{n} \mathrm{E}\left(\left[\int_{0}^{T}\left\|u_{n}(t, \cdot)\right\|_{q}^{a} \mathrm{~d} t\right]^{\beta}\right)<\infty .
$$

Using (1.12) with $q \geqslant 4$ and $\rho=q / 3$, so that $1 / r=1-2 / q$, we obtain

$$
\left\|H_{n}\left(u_{n}\right)(t, \cdot)\right\|_{q} \leqslant C \int_{0}^{t}(t-s)^{-(d+2) / 4+d /(4 r)}\left(\left\|u_{n}(s, \cdot)\right\|_{q}^{3}+1\right) \mathrm{d} s .
$$

Let $\left.\gamma, \gamma^{\prime} \in\right] 1,+\infty[$ be conjugate exponents, with $\gamma$ close enough to unity to ensure $(-(d+2) / 4+d /(4 r)) \gamma>-1$; then Hölder's inequality implies

$$
\left\|H_{n}\left(u_{n}\right)(t, \cdot)\right\|_{q} \leqslant C\left[\int_{0}^{t}(t-s)^{(-[d+2] / 4+[d /(4 r)]) \gamma} \mathrm{d} s\right]^{1 / \gamma}\left[\int_{0}^{t}\left(\left\|u_{n}(s, \cdot)\right\|_{q}^{3}+1\right)^{\gamma^{\prime}} \mathrm{d} s\right]^{1 / \gamma^{\prime}} .
$$

Using (2.34), we obtain 


$$
\sup _{n} \mathrm{E}\left(\sup _{t \in[0, T]} H_{n}\left(u_{n}\right)(t, \cdot) \|_{q}^{\beta}\right)<\infty
$$

(2.16), (2.17) and (2.35) imply that, for $\beta \in\left[q,+\infty[\right.$, and if $d=3$ for $\beta \in] q, 6 q /(6-q)^{+}[$ with $q \geqslant 4$,

$$
\sup _{n} \mathrm{E}\left(\sup _{t \in[0, T]}\left\|u_{n}(t, \cdot)\right\|_{q}^{\beta}\right)<\infty
$$

We can now conclude that $\tau_{\infty}=+\infty$ a.s.; indeed, for every $T>0$,

$$
P\left(\tau_{n} \leqslant T\right)=P\left(\sup _{t \leqslant T}\left\|u_{n}(t, \cdot)\right\|_{q} \geqslant n\right) \leqslant \mathrm{E}\left(\sup _{t \leqslant T}\left\|u_{n}(t, \cdot)\right\|_{q}^{2 \beta}\right) n^{-2 \beta},
$$

so that $\lim _{n \rightarrow \infty} P\left(\tau_{n} \leqslant T\right)=0$. Therefore, we can construct the solution to the SPDE (1.10) on every interval $[0, T]$; this completes the proof of Theorem 1.3.

\subsection{Path regularity of $u$}

We prove Theorem 1.4, and study separately each term on the right-hand side of (1.10). Let us prove two lemmas on the regularity of $G u_{0}$.

Lemma 2.1. If $u_{0}$ is continuous, the function $G . u_{0}(*)$ is continuous.

Proof. The function $G u_{0}$ is continuous on $[a, T] \times D$, for $a>0$, because the series which defines $G$ is absolutely convergent on $[a, T] \times D$. We just need to prove continuity at $t=0$. Let $x_{0} \in D$ be fixed; at $\epsilon>0$ given, there exists $\eta>0$ such that $\left|x_{0}-y\right|<\eta$ implies $\left|u_{0}(y)-u_{0}\left(x_{0}\right)\right|<\epsilon$. Using the fact that $G$ is a semigroup and (1.6), we find

$$
\begin{aligned}
\left|G_{t} u_{0}\left(x_{0}\right)-u_{0}\left(x_{0}\right)\right| & =\left|\int_{D} G\left(t, x_{0}, y\right)\left(u_{0}(y)-u_{0}(x)\right) \mathrm{d} y\right| \\
& \leqslant \epsilon \int_{\left|y-x_{0}\right| \leqslant \eta}\left|G\left(t, x_{0}, y\right)\right| \mathrm{d} y+M \int_{\left|y-x_{0}\right| \geqslant \eta}\left|G\left(t, x_{0}, y\right)\right| \mathrm{d} y \\
& \leqslant C \epsilon+C \int_{|z|>\eta t^{-1 / 4}} \exp \left(-z^{4 / 3}\right) \mathrm{d} z .
\end{aligned}
$$

The last integral on the right-hand side converges to 0 as $t$ tends to 0 ; this concludes the proof.

Lemma 2.2. If $u_{0}$ belongs to $\mathscr{C}^{\alpha}(D)$, for $0<\alpha<1$, the function $G . u_{0}(*)$ belongs to $\mathscr{C}^{\alpha / 4, \alpha}([0, T], D)$. 
Proof. The proof is inspired by Bally et al. (1995, Lemma A.2), in the case of the heat kernel. Let us study the time increment. Because $G$ is a semigroup, using (1.6) we find that

$$
\begin{aligned}
\left|G_{t} u_{0}(x)-G_{s} u_{0}(x)\right| & =\left|\int_{D} \int_{D} G(s, x, y) G(t-s, y, z) u_{0}(z) \mathrm{d} y \mathrm{~d} z-\int_{D} G(s, x, y) u_{0}(y) \mathrm{d} y\right| \\
& =\left|\int_{D} G(s, x, y)\left(\int_{D} G(t-s, y, z)\left(u_{0}(z)-u_{0}(y)\right) \mathrm{d} z\right) \mathrm{d} y\right| \\
& \leqslant C \int_{D}|G(s, x, y)| \int_{D} G(t-s, y, z) \| z-\left.y\right|^{\alpha} \mathrm{d} z \mathrm{~d} y \\
& \leqslant C \int_{D}|G(s, x, y) \| t-s|^{\alpha / 4} \mathrm{~d} y \\
& \leqslant C|t-s|^{\alpha / 4} .
\end{aligned}
$$

For the space variable, we prove the regularity with respect to the first space coordinate; the proof is similar for the other ones. If $d \geqslant 2$, we set $x=\left(x_{1}, \tilde{x}\right)$, where $\tilde{x}=\left(x_{2}, \ldots, x_{d}\right)$ and $D=[0, \pi] \times \tilde{D}$. With this notation, we find that

$$
G(t, x, y)=\sum_{k \in \mathbb{N}^{d}} \exp \left(-\lambda_{k}^{2} t\right) \varepsilon_{k_{1}\left(x_{1}\right)} \varepsilon_{\tilde{k}}(\tilde{x}) \varepsilon_{k_{1}\left(y_{1}\right)} \varepsilon_{\tilde{k}}(\tilde{y})
$$

Notice that

$$
\varepsilon_{k_{1}}\left(x_{1}\right) \varepsilon_{k_{1}}\left(y_{1}\right)=\frac{1}{2}\left[\varepsilon_{k_{1}}\left(x_{1}+y_{1}\right)+\varepsilon_{k_{1}}\left(x_{1}-y_{1}\right)\right]
$$

and hence

$$
G(t, x, y)=\phi_{t}\left(x_{1}+y_{1}, \tilde{x}, \tilde{y}\right)+\phi_{t}\left(x_{1}-y_{1}, \tilde{x}, \tilde{y}\right),
$$

with $\phi_{t}\left(x_{1}, \tilde{x}, \tilde{y}\right)=\frac{1}{2} G\left(t,\left(x_{1}, \tilde{x}\right),(0, \tilde{y})\right)$. Furthermore, $\phi_{t}\left(x_{1}+2 \pi, \tilde{x}, \tilde{y}\right)=\phi_{t}\left(x_{1}, \tilde{x}, \tilde{y}\right)=$ $\phi_{t}\left(-x_{1}, \tilde{x}, \tilde{y}\right)$ and

$$
\sup _{t \in[0, T]} \sup _{\left(x_{1}, \tilde{x}\right) \in D} \int_{D}\left|\phi_{T}\left(x_{1}, \tilde{x}, \tilde{y}\right)\right| \mathrm{d} y \leqslant C .
$$

Take $x=\left(x_{1}, \tilde{x}\right)$ and $x^{\prime}=\left(x_{1}^{\prime}, \tilde{x}\right)$, with $x_{1}>x_{1}^{\prime}$; we have 


$$
\begin{aligned}
G u_{0}(t, x)-G u_{0}\left(t, x^{\prime}\right)= & \int_{0}^{\pi} \int_{\tilde{D}}\left[\phi_{t}\left(x_{1}+y_{1}, \tilde{x}, \tilde{y}\right)+\phi_{t}\left(x_{1}-y_{1}, \tilde{x}, \tilde{y}\right)\right] u_{0}(y) \mathrm{d} \tilde{y} \mathrm{~d} y_{1} \\
& -\int_{0}^{\pi} \int_{\tilde{D}}\left[\phi_{t}\left(x_{1}^{\prime}+y_{1}, \tilde{x}, \tilde{y}\right)+\phi_{t}\left(x_{1}^{\prime}-y_{1}, \tilde{x}, \tilde{y}\right)\right] u_{0}(y) \mathrm{d} \tilde{y} \mathrm{~d} y_{1} \\
= & \int_{\left.x_{1}-x_{1}^{\prime}\right)}^{\pi+\left(x_{1}-x_{1}^{\prime}\right)} \int_{\tilde{D}} \phi_{t}\left(x_{1}^{\prime}+y_{1}, \tilde{x}, \tilde{y}\right) u_{0}\left(y_{1}-\left(x_{1}-x_{1}^{\prime}\right), \tilde{y}\right) \mathrm{d} \tilde{y} \mathrm{~d} y_{1} \\
& -\int_{0}^{\pi} \int_{\tilde{D}} \phi_{t}\left(x_{1}^{\prime}+y_{1}, \tilde{x}, \tilde{y}\right) u_{0}(y) \mathrm{d} \tilde{y} \mathrm{~d} y_{1} \\
& +\int_{-\left(x_{1}-x_{1}^{\prime}\right)}^{\pi-\left(x_{1}-x_{1}^{\prime}\right)} \int_{\tilde{D}} \phi_{t}\left(x_{1}^{\prime}-y_{1}, \tilde{x}, \tilde{y}\right) u_{0}\left(y_{1}+\left(x_{1}-x_{1}^{\prime}\right), \tilde{y}\right) \mathrm{d} \tilde{y} \mathrm{~d} y_{1} \\
& -\int_{0}^{\pi} \int_{\tilde{D}} \phi_{t}\left(x_{1}^{\prime}-y_{1}, \tilde{x}, \tilde{y}\right) u_{0}(y) \mathrm{d} \tilde{y} \mathrm{~d} y_{1} \\
= & \sum_{i=1}^{4} D_{i}\left(t, x, x^{\prime}\right),
\end{aligned}
$$

with

$$
\begin{aligned}
D_{1}\left(t, x, x^{\prime}\right)= & \int_{x_{1}-x_{1}^{\prime}}^{\pi} \int_{\tilde{D}} \phi_{t}\left(x_{1}^{\prime}+y_{1}, \tilde{x}, \tilde{y}\right)\left[u_{0}\left(y_{1}-\left(x_{1}-x_{1}^{\prime}\right), \tilde{y}\right)-u_{0}(y)\right] \mathrm{d} \tilde{y} \mathrm{~d} y_{1} \\
D_{2}\left(t, x, x^{\prime}\right)= & \int_{0}^{\pi-\left(x_{1}-x_{1}^{\prime}\right)} \int_{\tilde{D}} \phi_{t}\left(x_{1}^{\prime}-y_{1}, \tilde{x}, \tilde{y}\right)\left[u_{0}\left(y_{1}+\left(x_{1}-x_{1}^{\prime}\right), \tilde{y}\right)-u_{0}(y)\right] \mathrm{d} \tilde{y} \mathrm{~d} y_{1} \\
D_{3}\left(t, x, x^{\prime}\right)= & -\int_{0}^{\left(x_{1}-x_{1}^{\prime}\right)} \int_{\tilde{D}} \phi_{t}\left(x_{1}^{\prime}+y_{1}, \tilde{x}, \tilde{y}\right) u_{0}(y) \mathrm{d} \tilde{y} \mathrm{~d} y_{1} \\
& +\int_{-\left(x_{1}-x_{1}^{\prime}\right)}^{0} \int_{\tilde{D}} \phi_{t}\left(x_{1}^{\prime}-y_{1}, \tilde{x}, \tilde{y}\right) u_{0}\left(y_{1}+\left(x_{1}-x_{1}^{\prime}\right), \tilde{y}\right) \mathrm{d} \tilde{y} \mathrm{~d} y_{1} \\
D_{4}\left(t, x, x^{\prime}\right)= & \int_{\pi}^{\pi+\left(x_{1}-x_{1}^{\prime}\right)} \int_{\tilde{D}} \phi_{t}\left(x_{1}^{\prime}+y_{1}, \tilde{x}, \tilde{y}\right) u_{0}\left(y_{1}-\left(x_{1}-x_{1}^{\prime}\right), \tilde{y}\right) \mathrm{d} \tilde{y} \mathrm{~d} y_{1} \\
& -\int_{\pi-\left(x_{1}-x_{1}^{\prime}\right)}^{\pi} \int_{\tilde{D}} \phi_{t}\left(x_{1}^{\prime}-y_{1}, \tilde{x}, \tilde{y}\right) u_{0}(y) \mathrm{d} \tilde{y} \mathrm{~d} y_{1} .
\end{aligned}
$$

Using (2.38) and the fact that $u_{0}$ has $\alpha$-Hölder continuous trajectories, we obtain

$$
\left|D_{1}\left(t, x, x^{\prime}\right)\right|+\left|D_{2}\left(t, x, x^{\prime}\right)\right| \leqslant C\left|x_{1}-x_{1}^{\prime}\right|^{\alpha} .
$$

For $D_{3}$, we notice first that 


$$
D_{3}\left(t, x, x^{\prime}\right)=\int_{0}^{\left(x_{1}-x_{1}^{\prime}\right)} \int_{\tilde{D}} \phi_{t}\left(x_{1}^{\prime}+y_{1}, \tilde{x}, \tilde{y}\right)\left[-u_{0}(y)+u_{0}\left(-y_{1}+\left(x_{1}-x_{1}^{\prime}\right), \tilde{y}\right)\right] \mathrm{d} \tilde{y} \mathrm{~d} y_{1} ;
$$

the estimation is then similar to that of $D_{1}$ and $D_{2}$. For the last term, since $\phi_{t}$ is $2 \pi$-periodic, we have

$D_{4}\left(t, x, x^{\prime}\right)=\int_{0}^{\left(x_{1}-x_{1}^{\prime}\right)} \int_{\tilde{D}} \phi_{t}\left(\pi+x_{1}^{\prime}+y_{1}, \tilde{x}, \tilde{y}\right)\left[u_{0}\left(\pi+y_{1}-\left(x_{1}-x_{1}^{\prime}\right), \tilde{y}\right)-u_{0}\left(\pi-y_{1}, \tilde{y}\right)\right] \mathrm{d} \tilde{y} \mathrm{~d} y_{1}$,

which immediately yields the same upper estimate as that of $D_{1}$ and $D_{2}$.

Using Lemma 1.8, the fact that $\sigma$ is bounded and Kolmogorov's lemma, we see that the stochastic term is a.s. Hölder continuous; more precisely, it belongs to $\mathscr{C}^{\gamma, \gamma^{\prime}}([0, T], D)$, with $\gamma<(1-d / 4) / 2, \gamma^{\prime}<2-d / 2 \leqslant 1$.

We need to study the regularity of the drift term; for this, we use the factorization method (see, for example, Da Prato and Zabczyk 1992). We remark that

$$
\Delta G(t, x, y)=\int_{D} G(t-s, x, z) \Delta G(s, z, y) \mathrm{d} z .
$$

Fix $\alpha \in] 0,1[$ and set

$$
\begin{aligned}
& \mathscr{J}(v)(t, x)=\int_{0}^{t} \int_{D} G(t-s, x, z)(t-s)^{-\alpha} v(s, z) \mathrm{d} s \mathrm{~d} z, \\
& \mathscr{R}(v)(s, z)=\int_{0}^{s} \int_{D} \Delta G\left(s-s^{\prime}, z, y\right)\left(s-s^{\prime}\right)^{\alpha-1}\left(f\left(v\left(s^{\prime}, y\right)\right)\right) \mathrm{d} y \mathrm{~d} s^{\prime} ;
\end{aligned}
$$

then the drift term is $H(u)(t, x)=\pi^{-1} \sin (\pi \alpha) \mathscr{F}(\mathscr{K}(v))(t, x)$, for all $t, x \in[0, T] \times D$. Because $u_{0}$ is continuous, it belongs to all $L^{q}(D), q \geqslant 4>3 \vee 3 d / 2$, and $u$ belongs to $L^{\infty}\left([0, T], L^{q}(D)\right)$ a.s. according to Theorem 1.3. We first show that $\mathscr{6}$ is an operator mapping $L^{\infty}\left([0, T], L^{q}(D)\right)$ into itself. Using (1.15), with $q$ and $q / 3,1 / r=1-2 / q$, we obtain

$$
\|\mathscr{K}(v)(t, \cdot)\|_{q} \leqslant \int_{0}^{t}(t-s)^{-1+\alpha-(d+2) / 4+(d / 4)(1-2 / q)}\left(1+\|v(s, \cdot)\|_{q}^{3}\right) \mathrm{d} s .
$$

We require $-1+\alpha-1 / 2-d /(2 q)>-1$, that is, $\alpha>1 / 2+d /(2 q)$.

Then we study $\mathscr{J}$, and prove that $\mathscr{J}(v)$ is Hölder continuous if $v \in L^{\infty}\left([0, T], L^{q}(D)\right)$. Let us first study the regularity with respect to the space variable. For $x, x^{\prime} \in D$,

$$
\begin{aligned}
& A\left(t, x, x^{\prime}\right)=\int_{0}^{t} \int_{D} 1_{\left\{|x-y| \leqslant\left|x-x^{\prime}\right|\right\}}(t-s)^{-\alpha}\left(|G(t-s, x, y)|+\left|G\left(t-s, x^{\prime}, y \mid\right)\right| u(s, y) \mid \mathrm{d} y \mathrm{~d} s,\right. \\
& B\left(t, x, x^{\prime}\right)=\int_{0}^{t} \int_{D} 1_{\left\{|x-y|>\left|x-x^{\prime}\right|\right\}}(t-s)^{-\alpha}\left|G(t-s, x, y)-G\left(t-s, x^{\prime}, y\right)\right||u(s, y)| \mathrm{d} y \mathrm{~d} s
\end{aligned}
$$

then

$$
\left|\mathscr{J}(v)(t, x)-\mathscr{J}(v)\left(t, x^{\prime}\right)\right| \leqslant A\left(t, x, x^{\prime}\right)+B\left(t, x, x^{\prime}\right)
$$


Using Hölder's inequality with $q$ and its conjugate exponent, (1.6) and (1.14) we find, for $\beta \in] 0,4(1-\alpha-d /(4 q))[$, that

$$
A\left(t, x, x^{\prime}\right) \leqslant\left|x-x^{\prime}\right|^{\beta} \int_{0}^{t}(t-s)^{-\alpha-\beta / 4-d /(4 q)}\|u(s, \cdot)\|_{q} \mathrm{~d} s \leqslant C\left|x-x^{\prime}\right|^{\beta} .
$$

We notice that if $|x-y| \geqslant\left|x-x^{\prime}\right|$, and $\tilde{x}$ denotes a point between $x$ and $x^{\prime}$, then $|\tilde{x}-y| \geqslant 2^{-1 / 2}\left(\left|2 x-x^{\prime}-y\right| \wedge\left|x^{\prime}-y\right|\right)$. Let $\lambda \in[0,1]$; using Hölder's inequality, Taylor's formula, (1.7) and (1.14), we find that

$$
\begin{aligned}
B\left(t, x, x^{\prime}\right) \leqslant & \left|x-x^{\prime}\right|^{\lambda} \int_{0}^{t} \int_{D}(t-s)^{-\alpha}|G(t-s, x, y)|+\left|G\left(t-s, x^{\prime}, y\right)\right|^{1-\lambda} \\
& \times 1_{\left\{|x-y| \geqslant\left|x-x^{\prime}\right|\right\}}\left[\int_{0}^{1}\left|\partial_{x} G\left(t-s, \theta x+(1-\theta) x^{\prime}, y\right)\right| \mathrm{d} \theta\right]^{\lambda}|u(s, y)| \mathrm{d} y \mathrm{~d} s \\
\leqslant & C\left|x-x^{\prime}\right|^{\lambda} \int_{0}^{t} \int_{D}(t-s)^{-\alpha-d(1-\lambda) / 4-\lambda(d+1) / 4} \\
& \times\left[\exp \left(-\frac{c\left|2 x-x^{\prime}-y\right|^{4 / 3}}{|t-s|^{1 / 3}}\right)+\exp \left(-\frac{c\left|x^{\prime}-y\right|^{4 / 3}}{|t-s|^{1 / 3}}\right)\right]^{\lambda} u(s, y) \mathrm{d} y \mathrm{~d} s \\
\leqslant & C\left|x-x^{\prime}\right|^{\lambda} \int_{0}^{t}(t-s)^{-\alpha-d / 4-\lambda / 4+(d / 4)(1-1 / q)}\|u(s, \cdot)\|_{q} \mathrm{~d} s .
\end{aligned}
$$

We need $-\alpha-d /(4 q)-\lambda / 4>-1$, that is, $\lambda<4(1-\alpha-d /(4 q))$. Since $q$ can be chosen as big as we want, $\mathscr{J}(v)$ is $\lambda$-Hölder continuous with respect to the space variable with $\lambda<1$.

Let us study the time regularity; for $0 \leqslant t<t^{\prime} \leqslant T$, we have

$$
\begin{aligned}
& \mathscr{J}(v)\left(t^{\prime}, x\right)-\mathscr{J}(v)(t, x) \\
& =\int_{t}^{t^{\prime}} \int_{D} G\left(t^{\prime}-s, x, z\right)\left(t^{\prime}-s\right)^{-\alpha} v(s, z) \mathrm{d} s \mathrm{~d} z \\
& \quad+\int_{0}^{t} \int_{D}\left[G\left(t^{\prime}-s, x, z\right)\left(t^{\prime}-s\right)^{-\alpha}-G(t-s, x, z)(t-s)^{-\alpha}\right] v(s, z) \mathrm{d} s \mathrm{~d} z .
\end{aligned}
$$

Using (1.6) and Hölder's inequality with $q$ and its conjugate exponent and (1.14), we have, for $\alpha+d /(4 q)<1$,

$$
\begin{aligned}
\left|\int_{t}^{t^{\prime}} \int_{D} G\left(t^{\prime}-s, x, z\right)\left(t^{\prime}-s\right)^{-\alpha} v(s, z) \mathrm{d} s \mathrm{~d} z\right| & \leqslant \int_{t}^{t^{\prime}}\left(t^{\prime}-s\right)^{-\alpha-d /(4 q)}\|v(s, \cdot)\|_{q} \mathrm{~d} s \\
& \leqslant\left|t-t^{\prime}\right|^{1-\alpha-d /(4 q)} \sup _{s \in[0, T]}\|v(s, \cdot)\|_{q} .
\end{aligned}
$$

For the second term, let $\mu \in[0,1], \epsilon>0$; Taylor's formula, Hölder's inequality and the fact that if $\tilde{t} \in\left[t, t^{\prime}\right]$, then $|\tilde{t}-s|>|t-s|$ for $s \in[0, t]$, imply that 


$$
\begin{aligned}
& \left|\int_{0}^{t} \int_{D}\left[G\left(t^{\prime}-s, x, y\right)\left(t^{\prime}-s\right)^{-\alpha}-G(t-s, x, y)(t-s)^{-\alpha}\right] v(s, y) \mathrm{d} s \mathrm{~d} y\right| \\
& \quad \leqslant C\left|t-t^{\prime}\right|^{\mu} \int_{0}^{t} \int_{D}(t-s)^{-\alpha-[d(1-\mu)] / 4}|v(s, y)| \\
& \quad \times\left[\int_{0}^{1}\left(\theta t+(1-\theta) t^{\prime}-s\right)^{-(d+4) / 4} \exp \left(-c \frac{|x-y|^{4 / 3}}{\left|\theta t+(1-\theta) t^{\prime}-s\right|^{1 / 3}}\right) \mathrm{d} \theta\right]^{\mu} \mathrm{d} y \mathrm{~d} s \\
& \leqslant C\left|t-t^{\prime}\right|^{\mu} \int_{0}^{t} \int_{D}(t-s)^{-\alpha-d / 4-\mu+\epsilon}|x-y|^{-4 \epsilon}|v(s, y)| \mathrm{d} y \mathrm{~d} s \\
& \quad \leqslant C\left|t-t^{\prime}\right|^{\mu} \int_{0}^{t}(t-s)^{-\alpha-d / 4-\mu+\epsilon}\left\|(x-\cdot)^{-4 \epsilon}\right\|_{q / q-1}\|v(s, \cdot)\|_{q} \mathrm{~d} s \leqslant C\left|t-t^{\prime}\right|^{\mu},
\end{aligned}
$$

with $\mu<1-\alpha-d / 4+\epsilon$ and $\epsilon<d(1-1 / q) / 4$. Since $\alpha>1 / 2+d /(2 q)$, we obtain $\mu<1-\alpha-d /(4 q)<1 / 2-3 d /(4 q)$. Since $q$ can be chosen as big as we want, we conclude that the function $\mathscr{J}(v)$ is $\mu$-Hölder continuous in time with $\mu<\frac{1}{2}$. This concludes the proof of Theorem 1.4.

\subsection{Improvement of the regularity of $u$ when $d=1$}

We suppose here that $d=1$ and that Assumption $4^{\prime \prime}$ is satisfied. Let us again study the regularity of each term on the right-hand side of (1.10). As for the initial condition term, we prove the following result.

Lemma 2.3. If $u_{0}$ belongs to $\mathscr{C}^{2+\epsilon}(D)$, for $0 \leqslant \epsilon<1$, the function $G . u_{0}(*)$ belongs to $\mathscr{C}^{(2+\epsilon) / 4,2+\epsilon}([0, T], D)$.

Proof. We first notice that $U(t, x)=u_{0}(x)-\int_{0}^{t} \int_{D} \Delta_{x} G(s, x, y) u_{0}^{\prime \prime}(y) \mathrm{d} y \mathrm{~d} s \quad$ satisfies the partial differential equation $\partial_{t} U+\Delta^{2} U=0$ with the boundary conditions (1.2) and $U(0, \cdot)=u_{0}$; hence $U(t, x)=G_{t} u_{0}(x)$. Since $\int_{D} G(s, x, y) \mathrm{d} y=1, \int_{D} \Delta_{x} G(s, x, y) \mathrm{d} y=0$, and hence

$$
G_{t} u_{0}(x)-u_{0}(x)=\int_{0}^{t} \int_{D} \Delta_{x} G(s, x, y)\left(u_{0}^{\prime \prime}(y)-u_{0}^{\prime \prime}(x)\right) \mathrm{d} y \mathrm{~d} s
$$

Then using (1.7) and the hypothesis on $u_{0}$, we deduce that

$$
\left|G_{t} u_{0}(x)-u_{0}(x)\right| \leqslant C t^{(2+\epsilon) / 4} .
$$

Since $G_{t} u_{0}(x)-G_{s} u_{0}(x)=\int_{D} G(s, x, y)\left(G_{t-s} u_{0}(y)-u_{0}(y)\right) \mathrm{d} y$, we deduce the time regularity of $G u_{0}$. 
For the space regularity, we use (2.37) and notice that

$\Delta G_{t} u_{0}(x)=\int_{D}\left(\phi^{\prime \prime}(t, x+y)+\phi^{\prime \prime}(t, x-y)\right) u_{0}(y) \mathrm{d} y=\int_{D}(\phi(t, x+y)+\phi(t, x-y)) u_{0}^{\prime \prime}(y) \mathrm{d} y$

for $t>0$. Hence the proof of space regularity of $G u_{0}$ is similar to the corresponding one in Lemma 2.2, using the same decomposition.

Let us prove that the stochastic term $L(u)$ defined by (2.5) belongs to $\mathscr{C}^{\gamma, 4 \gamma}([0, T], D)$ with $\gamma<\frac{3}{8}$. This time regularity has already been established. It suffices to prove that $L(u)$ is differentiable with respect to the space variable, and that its derivative is $\lambda$-Hölder continuous with $\lambda<\frac{1}{2}$. We use the same methods as Kunita (1984, p. 219) and introduce $\eta(t, x, \xi)=(L(u)(t, x+\xi)-L(u)(t, x)) \xi^{-1}$ for $\left.\left.\xi \in\right] 0, \pi-x\right]$. We have to evaluate $\mathrm{E}\left(\left|\eta(t, x, \xi)-\eta\left(t, x^{\prime}, \xi^{\prime}\right)\right|^{p}\right)$. Using Burkholder's inequality and the fact that the sequence $\left(\varepsilon_{k}\right)_{k \in \mathbb{N}}$ is an orthonormal basis of $L^{2}(D)$ and that $\sigma$ is bounded, we obtain

$$
\begin{aligned}
\mathrm{E}\left(\left|\eta(t, x, \xi)-\eta\left(t, x^{\prime}, \xi^{\prime}\right)\right|^{p}\right) \leqslant & C\left[\int _ { 0 } ^ { t } \int _ { D } \left[(G(t-s, x+\xi, y)-G(t-s, x, y)) \xi^{-1}\right.\right. \\
& \left.\left.-\left(G\left(t-s, x^{\prime}+\xi^{\prime}, y\right)-G\left(t-s, x^{\prime}, y\right)\right)\left(\xi^{\prime}\right)^{-1}\right]^{2} \mathrm{~d} y \mathrm{~d} s\right]^{p / 2} \\
\leqslant & C\left[\int _ { 0 } ^ { t } \sum _ { k \in \mathbb { N } ^ { * } } \operatorname { e x p } ( - 2 ( t - s ) k ^ { 4 } ) \left[\{\cos (k(x+\xi))-\cos (k x)\} \xi^{-1}\right.\right. \\
& \left.\left.-\left\{\cos \left(k\left(x^{\prime}+\xi^{\prime}\right)\right)-\cos \left(k x^{\prime}\right)\right\}\left(\xi^{\prime}\right)^{-1}\right]^{2} \mathrm{~d} s\right]^{p / 2} .
\end{aligned}
$$

Using the identity $\cos (y+k \xi)-\cos (y)=-k \xi \int_{0}^{1} \sin (y+k \xi u) \mathrm{d} u$, a simple computation shows that, for $0 \leqslant \lambda \leqslant 1$,

$\mathrm{E}\left(\left|\eta(t, x, \xi)-\eta\left(t, x^{\prime}, \xi^{\prime}\right)\right|^{p}\right) \leqslant C\left[\int_{0}^{t} \sum_{k \in \mathbb{N}^{*}} \exp \left(-2(t-s) k^{4}\right) k^{2+2 \lambda}\left(\left|x-x^{\prime}\right|+\left|\xi-\xi^{\prime}\right|\right)^{2 \lambda} \mathrm{d} s\right]^{p / 2}$.

Thus, for $\lambda<\frac{1}{2}$, we finally obtain that

$$
\mathrm{E}\left(\left|\eta(t, x, \xi)-\eta\left(t, x^{\prime}, \xi^{\prime}\right)\right|^{p}\right) \leqslant C\left(\left|x-x^{\prime}\right|+\left|\xi-\xi^{\prime}\right|\right)^{\lambda p} .
$$

Therefore, $\eta(t, x, \cdot)$ can be extended as a continuous function on $[0, \pi]$, and $\eta(t, \cdot, 0)$ is the space derivative of $L(u)(t, x)$ which also has $\lambda$-Hölder continuous trajectories.

For the last term $H(u)$, we proceed in the same way as in the preceding subsection. We observe that $\partial_{x}^{3} G(t, x, z)=\int_{D} \partial_{x}^{3} G(t-s, x, y) G(s, y, z) \mathrm{d} y$, and replace in the function $\mathscr{K}$ defined by (2.40) the second-order derivative by the third-order one. Using the factorization method again, we prove that the space derivative of $H(u)$ is $\lambda$-Hölder with $\lambda<1$, so that $H(u)$ belongs to $\mathscr{C}^{\mu / 4, \mu}([0, T], D)$ with $\mu<\frac{3}{2}$. 


\section{Malliavin calculus for the Cahn-Hilliard SPDE}

In this section we suppose that Assumption 4 holds, because we need $u$ to be continuous. We first prove local regularity of $u$ in the sense of the Malliavin calculus. Let us recall some classical notation of Malliavin calculus (see Nualart 1995). $\mathbb{D}^{1,2}$ is the set of random variables $F$ such that the Malliavin derivative $D F$ exists and satisfies

$$
\|F\|_{1,2}=\left[\mathrm{E}\left(|F|^{2}\right)+\mathrm{E}\left(\|D F\|_{L^{2}([0, T] \times D)}^{2}\right)\right]^{1 / 2}<\infty .
$$

A process $X \in L^{2}(\Omega \times[0, T] \times D)$ belongs to $\mathbb{L}^{1,2}$ if, for each $(s, x) \in[0, T] \times D, X_{s, x}$ belongs to $\mathbb{D}^{1,2}$ and

$$
\mathrm{E}\left(\int_{0}^{T} \int_{D} \int_{0}^{T} \int_{D}\left|D_{s, y} X(t, x)\right|^{2} \mathrm{~d} y \mathrm{~d} s \mathrm{~d} x \mathrm{~d} t\right)<+\infty .
$$

We define the 'local' versions $\mathbb{D}_{\text {loc }}^{1,2}$ and $\mathbb{L}_{\text {loc }}^{1,2}$ of these two spaces as follows. $X$ belongs to $\mathbb{D}_{\text {loc }}^{1,2}$ $\left(\mathbb{L}_{\text {loc }}^{1,2}\right)$ if there exists a sequence $\Omega_{n}$ such that, for every $n, X=X_{n}$ on $\Omega_{n}$ a.s., $X_{n}$ belong to $\mathbb{Q}_{\text {loc }}^{1,2}\left(\mathbb{L}_{\text {loc }}^{1,2}\right)$, and $\lim _{n \rightarrow \infty} P\left(\Omega_{n}\right)=1$.

Lemma 3.1. The solution $u$ of the SPDE (1.10) belongs to $\mathbb{L}_{\text {loc }}^{1,2}$.

\subsection{Approximation of $u$ by a sequence of elements of $\mathbb{L}^{1,2}$}

For every $n>0$, let us denote by

$$
\Omega_{n}=\left\{\omega \in \Omega\left|\sup _{0 \leqslant t \leqslant T} \sup _{x \in D}\right| u(t, x, w) \mid \leqslant n\right\} .
$$

Because the process $u$ is a.s. continuous, $\lim _{n \rightarrow \infty} P\left(\Omega_{n}\right)=1$. Let us construct an approximation $\left(u_{n}\right)_{n \in \mathbb{N}}$ of $u$, such that $u_{n}=u$ on $\Omega_{n}$ a.s. For this we still truncate the polynomial $f$, but in another way. Set $f_{n}(x)=K_{n}(|x|) f(x)$, where $K_{n}$ is defined by $(2.1) ; f_{n}$ is $\mathscr{C}^{1}$ function with bounded derivative. Let us denote by $u_{n}$ the solution to the SPDE

$$
\begin{aligned}
u_{n}(t, x)= & \int_{D} G(t, x, y) u_{0}(y) \mathrm{d} y+\int_{0}^{t} \int_{D} \Delta G(t-s, x, y) f_{n}(u(s, y)) \mathrm{d} y \mathrm{~d} s \\
& +\int_{0}^{t} \int_{D} G(t-s, x, y) \sigma\left(u_{n}(s, y)\right) W(\mathrm{~d} y, \mathrm{~d} s) .
\end{aligned}
$$

Notice that since $f_{n}$ is globally Lipschitz, a standard argument shows that (3.2) has a unique solution. The local property of stochastic integrals proves that $u=u_{n}$ on $\Omega_{n}$ a.s. In order to prove Lemma 3.1, according to Nualart $(1995$, p. 45), it suffices to check the following result.

Lemma 3.2. The solution $u_{n}$ to (3.2) exists and is unique; furthermore, it belongs to $\mathbb{1}^{1,2}$ and its Malliavin derivative satisfies the SPDE 


$$
\begin{aligned}
D_{s, y} u_{n}(t, x)= & G(t-s, x, y) \sigma\left(u_{n}(s, y)\right)+\int_{s}^{t} \int_{D} \Delta G(t-\theta, x, \eta) f_{n}^{\prime}\left(u_{n}(\theta, \eta)\right) D_{s, y} u_{n}(\theta, \eta) \mathrm{d} \eta \mathrm{d} \theta \\
& +\int_{s}^{t} \int_{D} G(t-\theta, x, \eta) S_{n}(\theta, \eta) D_{s, y} u_{n}(\theta, \eta) W(\mathrm{~d} \eta, \mathrm{d} \theta)
\end{aligned}
$$

if $s \leqslant t$, and $D_{s, y} u_{n}(t, x)=0$ if $s>t$, where $S_{n}(\theta, \eta)$ is $\mathscr{F}_{\theta}$-adapted, bounded and satisfies

$$
D_{s, y}\left(\sigma\left(u_{n}(\theta, \eta)\right)\right)=S_{n}(\theta, \eta) D_{s, y} u_{n}(\theta, \eta) .
$$

Proof. To prove the existence and the uniqueness of the solution to (3.3) we construct a Cauchy sequence $\left(u_{n, k}\right)_{k \in \mathbb{N}}$ converging to $u_{n}$ by the Picard iteration scheme, which means that

$$
u_{n, 0}(t, x)=G_{t} u_{0}(x)
$$

and

$$
\begin{aligned}
u_{n, k+1}(t, x)= & \int_{D} G(t, x, y) u_{0}(y) \mathrm{d} y+\int_{0}^{t} \int_{D} \Delta G(t-s, x, y) f_{n}\left(u_{n, k}(s, y)\right) \mathrm{d} y \mathrm{~d} s \\
& +\int_{0}^{t} \int_{D} G(t-s, x, y) \sigma\left(u_{n, k}(s, y)\right) W(\mathrm{~d} y, \mathrm{~d} s)
\end{aligned}
$$

for $k \geqslant 0$. Then for $k \geqslant 1$,

$$
\begin{aligned}
u_{n, k+1}(t, x)-u_{n, k}(t, x)= & \int_{0}^{t} \int_{D} \Delta G(t-s, x, y)\left[f_{n}\left(u_{n, k}(s, y)\right)-f_{n}\left(u_{n, k-1}(s, y)\right)\right] \mathrm{d} y \mathrm{~d} s \\
& +\int_{0}^{t} \int_{D} G(t-s, x, y)\left[\sigma\left(u_{n, k}(s, y)\right)-\sigma\left(u_{n, k-1}(s, y)\right)\right] W(\mathrm{~d} y, \mathrm{~d} s) .
\end{aligned}
$$

Burkholder's inequality and the fact that $\sigma$ and $f_{n}$ are Lipschitz functions imply, for $p \in[2,+\infty[$, that

$$
\begin{aligned}
& \mathrm{E}\left(\left|u_{n, k+1}(t, x)-u_{n, k}(t, x)\right|^{p}\right) \\
& \leqslant C_{n} \mathrm{E}\left(\left|\int_{0}^{t} \int_{D}\right| \Delta G(t-s, x, y) \| u_{n, k}(s, y)-u_{n, k-1}(s, y)|\mathrm{d} y \mathrm{~d} s|^{p}\right) \\
& \quad+C \mathrm{E}\left(\left|\int_{0}^{t} \int_{D}\right| G^{2}(t-s, x, y) \| u_{n, k}(s, y)-\left.\left.u_{n, k-1}(s, y)\right|^{2} \mathrm{~d} y \mathrm{~d} s\right|^{p / 2}\right) .
\end{aligned}
$$

Lemma 1.6 applied twice with $q=\rho=\infty$ and $r=1$, and Hölder's inequality, imply that 


$$
\begin{aligned}
\mathrm{E}\left(\sup _{x \in D}\left|u_{n, k+1}(t, x)-u_{n, k}(t, x)\right|^{p}\right) \\
\leqslant C_{n} \mathrm{E}\left(\left|\int_{0}^{t}(t-s)^{-(d+2) / 4+d / 4}\left\|u_{n, k}(s, \cdot)-u_{n, k-1}(s, \cdot)\right\|_{\infty} \mathrm{d} s\right|^{p}\right) \\
+C \mathrm{E}\left(\left|\int_{0}^{t}(t-s)^{-d / 2+d / 4}\left\|u_{n, k}(s, \cdot)-u_{n, k-1}(s, \cdot)\right\|_{\infty}^{2} \mathrm{~d} s\right|^{p / 2}\right) \\
\leqslant C_{n} \mathrm{E}\left(\int_{0}^{t}(t-s)^{-[(d+2) \vee(2 d)] / 4+d / 4}\left\|u_{n, k}(t, \cdot)-u_{n, k-1}(t, \cdot)\right\|_{\infty}^{p} \mathrm{~d} s\right) .
\end{aligned}
$$

Let $b=\frac{1}{4}\{(d+2) \vee(2 d)\}$; iterating this inequality and using Fubini's theorem as in Walsh (1986, Lemma 3.3), we obtain

$$
\begin{aligned}
& \mathrm{E}\left(\left\|u_{n, k+2}(t, \cdot)-u_{n, k+1}(t, \cdot)\right\|_{\infty}^{p}\right) \\
& \quad \leqslant C_{n} \int_{0}^{t}(t-s)^{-b+(d / 4)} \mathrm{d} s \int_{0}^{s}(s-\tau)^{-b+d / 4} \mathrm{E}\left(\mid u_{n, k}(\tau, \cdot)-u_{n, k-1}(\tau, \cdot) \|_{\infty}^{p}\right) \mathrm{d} \tau \\
& \quad \leqslant C_{n} \int_{0}^{t}\left(\int_{\tau}^{t}(t-s)^{-b+d / 4}(s-\tau)^{-b+d / 4} \mathrm{~d} s\right) \mathrm{E}\left(\left\|u_{n, k}(\tau, \cdot)-u_{n, k-1}(\tau, \cdot)\right\|_{\infty}^{p}\right) \mathrm{d} \tau \\
& \quad \leqslant C_{n} \int_{0}^{t} \sup _{x \in D} \mathrm{E}\left(\left\|u_{n, k}(\tau, \cdot)-u_{n, k-1}(\tau, \cdot)\right\|_{\infty}^{p}\right) \mathrm{d} \tau .
\end{aligned}
$$

This implies, for $k \geqslant 0$, that

$$
\begin{gathered}
\sup _{t \in[0, T]} \mathrm{E}\left(\left\|u_{n, 2(k+1)}(t, \cdot)-u_{n, 2 k+1}(t, \cdot)\right\|_{\infty}^{p}\right) \leqslant \frac{\left(C_{n}\right)^{k}}{k !} \sup _{t \in[0, T]} \mathrm{E}\left(\left\|u_{n, 2}(t, \cdot)-u_{n, 1}(t, \cdot)\right\|_{\infty}^{p}\right) \\
\sup _{t \in[0, T]} \mathrm{E}\left(\left\|u_{n, 2 k+1}(t, \cdot)-u_{n, 2 k}(t, \cdot)\right\|_{\infty}^{p}\right) \leqslant \frac{\left(C_{n}\right)^{k}}{k !} \sup _{t \in[0, T]} \mathrm{E}\left(\left\|u_{n, 1}-u_{n, 0}\right\|_{\infty}^{p}\right) .
\end{gathered}
$$

Hence

$$
\sum_{k>0} \sup _{t \in[0, T]} \mathrm{E}\left(\left\|u_{n, k}(t, \cdot)-u_{n, k-1}(t, \cdot)\right\|_{\infty}^{p}\right)<\infty
$$

and the sequence $u_{n, k}(t, x)$ converges in $L^{p}(\Omega)$ as $k \rightarrow+\infty$, for $(t, x) \in[0, T] \times D$, to the solution to the SPDE (3.2) such that

$$
\sup _{t \in[0, T]} \mathrm{E}\left(\left\|u_{n}(t, \cdot)\right\|_{\infty}^{p}\right)<\infty
$$

for $p \in\left[2,+\infty\left[\right.\right.$. Computations similar to preceding ones shows that if $u_{n}$ and $v_{n}$ are solutions to (3.2), for $p \in[2,+\infty[$, 


$$
\sup _{t \in[0, T]} \mathrm{E}\left(\left\|u_{n}(t, \cdot)-v_{n}(t, \cdot)\right\|_{\infty}^{p}\right)<C_{n} \int_{0}^{t} \mathrm{E}\left(\left\|u_{n}(s, \cdot)-v_{n}(s, \cdot)\right\|_{\infty}^{p}\right) \mathrm{d} s .
$$

Gronwall's lemma implies $u_{n}(t, x)=v_{n}(t, x)$ a.s. Since the solution is a.s. continuous, the processes $u_{n}$ and $v_{n}$ are indistinguishable.

We now prove by induction that the sequence $u_{n, k}(t, x)$ belongs to $\mathbb{D}^{1,2}$. Since $u_{n, 0}$ is deterministic, it belongs to $\mathbb{D}^{1,2}$ and $D u_{n, 0} \equiv 0$. Suppose that, for $k \geqslant 0$ and for every $(t, x) \in[0, T] \times D, u_{n, k}(t, x)$ belongs to $\mathbb{D}^{1,2}$ and satisfies

$$
\sup _{t \in[0, T]} \sup _{x \in D} \mathrm{E}\left(\int_{0}^{T} \int_{D}\left|D_{s, y} u_{n, k}(t, x)\right|^{2} \mathrm{~d} y \mathrm{~d} s\right)<\infty .
$$

According to Nualart (1995, Proposition 1.2.3), since $\sigma$ is Lipschitz, there exists $S_{n, k}(\theta, \eta)$ such that

$$
D_{s, y}\left(\sigma\left(u_{n, k}(\theta, \eta)\right)\right)=S_{n, k}(\theta, \eta) D_{s, y} u_{n, k}(\theta, \eta)
$$

and

$$
\sup _{k, n, \theta, \eta}\left|S_{n, k}(\theta, \eta)\right|=C_{\sigma}<\infty .
$$

Let us take the Malliavin derivative of both sides of (3.4); then for $s \leqslant t$,

$D_{s, y} u_{n, k+1}(t, x)$

$$
\begin{aligned}
& =G(t-s, x, y) \sigma\left(u_{n, k}(s, y)\right)+\int_{s}^{t} \int_{D} \Delta G(t-\theta, x, \eta) f_{n}^{\prime}\left(u_{n, k}(\theta, \eta)\right) D_{s, y} u_{n, k}(\theta, \eta) \mathrm{d} \eta \mathrm{d} \theta \\
& +\int_{s}^{t} \int_{D} G(t-\theta, x, \eta) S_{n, k}(\theta, \eta) D_{s, y} u_{n, k}(\theta, \eta) W(\mathrm{~d} \eta, \mathrm{d} \theta)
\end{aligned}
$$

and for $s>t D_{s, y} u_{n, k+1}(t, x)=0$. We need to verify that

$$
\sup _{k} \sup _{t \in[0, T]} \sup _{x \in D} \mathrm{E}\left(\int_{0}^{T} \int_{D}\left|D_{s, y} u_{n, k}(t, x)\right|^{2} \mathrm{~d} y \mathrm{~d} s\right)<\infty .
$$

Clearly, $\mathrm{E}\left(\int_{0}^{T} \int_{D}\left|D_{s, n} u_{n, k}(t, x)\right|^{2} \mathrm{~d} y \mathrm{~d} s\right) \leqslant 3 \sum_{i=1}^{3} A_{i}(t, x)$, where

$$
\begin{aligned}
& A_{1}(t, x)=\mathrm{E}\left(\int_{0}^{T} \int_{D}\left|G(t-s, x, y) \sigma\left(u_{n, k}(s, y)\right)\right|^{2} \mathrm{~d} y \mathrm{~d} s\right), \\
& A_{2}(t, x)=\mathrm{E}\left(\int_{0}^{T} \int_{D}\left|\int_{s}^{t} \int_{D} \Delta G(t-\theta, x, \eta) f_{n}^{\prime}\left(u_{n, k}(\theta, \eta)\right) D_{s, y} u_{n, k}(\theta, \eta) \mathrm{d} \eta \mathrm{d} \theta\right|^{2} \mathrm{~d} y \mathrm{~d} s\right), \\
& A_{3}(t, x)=\mathrm{E}\left(\int_{0}^{T} \int_{D}\left|\iint_{s}^{t} \int_{D} G(t-\theta, x, \eta) S_{n, k}(\theta, \eta) D_{s, y} u_{n, k}(\theta, \eta) W(\mathrm{~d} \eta, \mathrm{d} \theta)\right|^{2} \mathrm{~d} y \mathrm{~d} s\right) .
\end{aligned}
$$

Since $\sigma$ is bounded, (1.18) implies that 


$$
\begin{aligned}
\sup _{k} \sup _{t \in[0, T]} \sup _{x \in D} A_{1}(t, x) & \leqslant\|\sigma\|_{\infty}^{2} \sup _{t \in[0, T]} \sup _{x \in D} \mathrm{E}\left(\int^{T} \int_{D} G^{2}(t-s, x, y) \mathrm{d} y \mathrm{~d} s\right) \\
& =C<\infty .
\end{aligned}
$$

Since $\left|f_{n}^{\prime}\right| \leqslant C_{n}$, Fubini's theorem and Hölder's inequality with respect to the measure $|\Delta G(t-\theta, x, \eta)| \mathrm{d} \eta \mathrm{d} \theta$ yield

$$
\begin{aligned}
A_{2}(t, x) & \leqslant C_{n} \int_{0}^{t} \int_{D}|\Delta G(t-\theta, x, \eta)| \mathrm{E}\left(\int_{0}^{\theta} \int_{D}\left|D_{s, y} u_{n, k}(\theta, \eta)\right|^{2} \mathrm{~d} y \mathrm{~d} s\right) \mathrm{d} \eta \mathrm{d} \theta \\
& \leqslant C_{n} \int_{0}^{t} \int_{D}|\Delta G(t-\theta, x, \eta)| \mathrm{d} \eta \sup _{\eta^{\prime}} \mathrm{E}\left(\int_{0}^{\theta} \int_{D}\left|D_{s, y} u_{n, k}\left(\theta, \eta^{\prime}\right)\right|^{2} \mathrm{~d} y \mathrm{~d} s\right) \mathrm{d} \theta .
\end{aligned}
$$

Thus (1.7) implies that

$$
A_{2}(t, x) \leqslant C \int_{0}^{t} \frac{1}{\sqrt{t-\theta}} \sup _{\eta} \mathrm{E}\left(\int_{0}^{\theta} \int_{D}\left|D_{s, y} u_{n, k}(\theta, \eta)\right|^{2} \mathrm{~d} y \mathrm{~d} s\right) \mathrm{d} \theta .
$$

Inequality (3.6), Burkholder's inequality, Fubini's theorem and (1.6) imply that

$$
\begin{aligned}
A_{3}(t, x) & \leqslant C \mathrm{E}\left(\int_{0}^{t} \int_{D} \int_{s}^{t} \int_{D}|G(t-\theta, x, \eta)|^{2}\left|D_{s, y} u_{n, k}(\theta, \eta)\right|^{2} \mathrm{~d} \eta \mathrm{d} \theta \mathrm{d} y \mathrm{~d} s\right) \\
& \leqslant C \int_{0}^{t}(t-\theta)^{-d / 4} \sup _{\eta} \mathrm{E}\left(\int_{0}^{\theta} \int_{D}\left|D_{s, y} u_{n, k}(\theta, \eta)\right|^{2} \mathrm{~d} y \mathrm{~d} s\right) \mathrm{d} \theta .
\end{aligned}
$$

Therefore, (3.9)-(3.11) yield the existence of positive constants $C$ and $C_{n}$ such that, for every $t \in[0, T]$ and $k \geqslant 0$,

$$
\begin{aligned}
& \sup _{x \in D} \mathrm{E}\left(\int_{0}^{t} \int_{D}\left|D_{s, y} u_{n, k+1}(t, x)\right|^{2} \mathrm{~d} y \mathrm{~d} s\right) \\
& \quad \leqslant C+C_{n} \int_{0}^{t}(t-\theta)^{-(1 / 2 \vee d / 4)} \sup _{\eta} \mathrm{E}\left(\int_{0}^{\theta} \int_{D}\left|D_{s, n} u_{n, k}(\theta, \eta)\right|^{2} \mathrm{~d} y \mathrm{~d} s\right) \mathrm{d} \theta ;
\end{aligned}
$$

therefore, iterating this inequality and using the convergence of the integral $\int_{s}^{t}(t-\theta)^{-(1 / 2 \vee d / 4)}(\theta-s)^{-(1 / 2 \vee d / 4)} \mathrm{d} \theta$, we deduce (3.8).

We have proved that $u_{n, k}(t, x)$ belongs to $\mathbb{D}^{1,2}$ for all $(t, x) \in[0, T] \times D$. Using Nualart (1995, Lemma 1.2.3), we deduce that the random variable $u_{n}(t, x)$ belongs to $\mathbb{D}^{1,2}$, and that the sequence $D u_{n, k}(t, x)$ converges to $D u_{n}(t, x)$ in the weak topology of $L^{2}([0, T] \times D \times \Omega)$. Let us define $S_{n}(\theta, \eta)$ as the weak limit of $\left(S_{n, k}(\theta, \eta)\right) k \geqslant 0$ in $L^{\infty}(\Omega \times[0, T] \times D)$; then $D_{s, y}\left(\sigma\left(u_{n}(\theta, \eta)\right)\right)=S_{n}(\theta, \eta) D_{s, y} u_{n}(\theta, \eta)$ and

$$
\sup _{n, \theta, \eta}\left|S_{n}(\theta, \eta)\right|=C_{\sigma}<\infty \text {. }
$$


Differentiating the $\operatorname{SPDE}(2.2)$, we obtain that for each $(t, x)$, the process $D_{s, y} u_{n}(t, x)$ satisfies the following SPDE:

$$
\begin{aligned}
V_{s, y}^{n}(t, x)= & G(t-s, x, y) \sigma\left(u_{n}(s, y)\right)+\int_{s}^{t} \int_{D} \Delta G(t-\theta, x, \eta) f_{n}^{\prime}\left(u_{n}(\theta, \eta)\right) V_{s, y}^{n}(\theta, \eta) \mathrm{d} \eta \mathrm{d} \theta \\
& +\int_{s}^{t} \int_{D} G(t-\theta, x, \eta) S_{n}(\theta, \eta) V_{s, y}^{n}(\theta, \eta) W(\mathrm{~d} \eta, \mathrm{d} \theta)
\end{aligned}
$$

for $s \leqslant t$ and $V_{s, y}^{n}(t, x)=0$ if $s>t$. We need to prove the uniqueness of the solution to (3.13). Let $V_{n}$ and $U_{n}$ be two solutions of (3.13). Computations similar to those made to prove (3.9)-(3.11) imply that

$$
\begin{aligned}
& \sup _{x \in D} \mathrm{E}\left(\int_{0}^{t} \int_{D}\left|V_{s, y}^{n}(t, x)-U_{s, y}^{n}(t, x)\right|^{2} \mathrm{~d} y \mathrm{~d} s\right) \\
& \quad \leqslant C_{n} \int_{0}^{t}(t-\theta)^{-\left(\frac{1}{2} \vee \frac{d}{4}\right)} \times \sup _{x \in D} \mathrm{E}\left(\int_{0}^{\theta} \int_{D}\left|V_{s, y}^{n}(\theta, x)-U_{s, y}^{n}(\theta, x)\right|^{2} \mathrm{~d} y \mathrm{~d} s \mathrm{~d} \theta\right),
\end{aligned}
$$

and Gronwall's generalized lemma yields

$$
\sup _{t \in[0, T]} \sup _{x \in D} \mathrm{E}\left(\int_{0}^{T} \int_{D}\left|V_{s, y}^{n}(t, x)-U_{s, y}^{n}(t, x)\right|^{2} \mathrm{~d} y \mathrm{~d} s\right)=0 .
$$

Hence (3.8) shows that the process $u_{n}$ belongs to $\mathbb{L}^{1,2}$, which implies that

$$
\sup _{t \in[0, T]} \sup _{x \in D} \mathrm{E}\left(\int_{0}^{t} \int_{D}\left|D_{s, y} u_{n}(t, x)\right|^{2} \mathrm{~d} y \mathrm{~d} s\right)=C_{n}<\infty
$$

this concludes the proof of Lemma 3.2.

\subsection{Existence of a density for the random variable $u_{n}(t, x)$}

Let $t>0$ and $x \in] 0, \pi\left[{ }^{d}\right.$; according to Nualart (1995, Theorem 2.1.3), we have to prove that

$$
\int_{0}^{t} \int_{D}\left|D_{r, z} u_{n}(t, x)\right|^{2} \mathrm{~d} r \mathrm{~d} z>0 \text { a.s. }
$$

Let us prove the following technical result for time integrals over small time intervals:

Lemma 3.3. There exists a constant $C_{n}$ such that, for every $0<\epsilon<t$,

$$
\sup _{s \in[t-\epsilon, t]} \sup _{y \in D} \mathrm{E}\left(\int_{t-\epsilon}^{t} \int_{D}\left|D_{r, z} u_{n}(s, y)\right|^{2} \mathrm{~d} z \mathrm{~d} r\right) \leqslant C_{n} \epsilon^{1-d / 4} .
$$

Proof. For $t-\epsilon \leqslant s \leqslant t$, set $H_{n}^{\epsilon}(s, y)=\mathrm{E}\left(\int_{t-\epsilon}^{s} \int_{D}\left|D_{r, z} u_{n}(s, y)\right|^{2} \mathrm{~d} z \mathrm{~d} r\right)$; (3.14) shows that 


$$
\sup _{s \in[0, T]} \sup _{y \in D} H_{n}^{\epsilon}(s, y)=C_{n}<\infty
$$

According to (3.2),

$$
H_{n}^{\epsilon}(s, y) \leqslant C \sum_{i=1}^{3} A_{i}(s, y, \epsilon)
$$

with

$$
\begin{aligned}
& A_{1}(s, y, \epsilon)=\mathrm{E}\left(\int_{t-\epsilon}^{s} \int_{D}\left|G(s-r, y, z) \sigma\left(u_{n}(r, z)\right)\right|^{2} \mathrm{~d} z \mathrm{~d} r\right) \\
& A_{2}(s, y, \epsilon)=\mathrm{E}\left(\int_{t-\epsilon}^{s} \int_{D}\left|\int_{r}^{s} \int_{D} \Delta G(s-\theta, x, \eta) f_{n}^{\prime}\left(u_{n}(\theta, \eta)\right) D_{r, z} u_{n}(\theta, \eta) \mathrm{d} \eta \mathrm{d} \theta\right|^{2} \mathrm{~d} r \mathrm{~d} z\right) \\
& A_{3}(s, x, \epsilon)=\mathrm{E}\left(\int_{t-\epsilon}^{s} \int_{D}\left|\int_{r}^{s} \int_{D} G(s-\theta, x, \eta) S_{n}(\theta, \eta) D_{r, z} u_{n}(\theta, \eta) W(\mathrm{~d} \eta, \mathrm{d} \theta)\right|^{2} \mathrm{~d} r \mathrm{~d} z\right)
\end{aligned}
$$

Because $\sigma$ is bounded, (1.6) implies that

$$
A_{1}(s, y, \epsilon) \leqslant C \epsilon^{1-d / 4} \text {. }
$$

Burkholder's inequality, Fubini's theorem, inequalities (1.6), (3.12), (3.17) and equation (1.14) imply that

$$
\begin{aligned}
A_{3}(s, y, \epsilon) & \leqslant C \mathrm{E}\left(\int_{t-\epsilon}^{s} \int_{D} \int_{r}^{s} \int_{D} G^{2}(s-\theta, y, \eta)\left|D_{r, z} u_{n}(\theta, \eta)\right|^{2} \mathrm{~d} \theta \mathrm{d} \eta \mathrm{d} z \mathrm{~d} r\right) \\
& \leqslant C \mathrm{E}\left(\int_{t-\epsilon}^{s} \int_{D} \int_{t-\epsilon}^{\theta} \int_{D} G^{2}(s-\theta, y, \eta)\left|D_{r, z} u_{n}(\theta, \eta)\right|^{2} \mathrm{~d} z \mathrm{~d} r \mathrm{~d} \eta \mathrm{d} \theta\right) \\
& \leqslant C \int_{t-\epsilon}^{s} \int_{D} G^{2}(s-\theta, y, \eta) H_{n}^{\epsilon}(\theta, \eta) \mathrm{d} \eta \mathrm{d} \theta \\
& \leqslant \int_{t-\epsilon}^{s}(s-\theta)^{-d / 4} \sup _{\eta \in D} H_{n}^{\epsilon}(\theta, \eta) \mathrm{d} \theta .
\end{aligned}
$$

For the other term, Schwarz's inequality with respect to the measure $|\Delta G(s-\theta, y, \eta)| \mathrm{d} \theta \mathrm{d} \eta$ implies that

$$
A_{2}(s, x, \epsilon) \leqslant C_{n} \mathrm{E}\left(\int_{t-\epsilon}^{s} \int_{D} \int_{r}^{s} \int_{D}\left|\Delta G_{s-\theta}(y, \eta) \| D_{r, z} u_{n}(\theta, \eta)\right|^{2} \mathrm{~d} \theta \mathrm{d} \eta \mathrm{d} z \mathrm{~d} r\right) .
$$

Using (1.7), by a computation similar to that of $A_{3}$, we find

$$
A_{2}(s, y, \epsilon) \leqslant C_{n} \int_{t-\epsilon}^{s}(s-\theta)^{-1 / 2} \sup _{\eta \in D} H_{n}^{\epsilon}(\theta, \eta) \mathrm{d} \theta .
$$


Inequalities (3.18)-(3.20) imply that

$$
\sup _{y \in D} H_{n}^{\epsilon}(s, y) \leqslant C \epsilon^{1-d / 4}+C_{n} \int_{t-\epsilon}^{s}(s-\theta)^{-(d / 4 \vee 1 / 2)} \sup _{\eta \in D} H_{n}^{\epsilon}(\theta, \eta) \mathrm{d} \theta
$$

Gronwall's generalized lemma concludes the proof of (3.16).

Let us prove Theorem 1.5 when $\sigma=1$.

Lemma 3.4. If $\sigma=1$, denote by $u_{n}$ the solution to (3.2). For $t>0$ and $\left.x \in\right] 0, \pi\left[{ }^{d}\right.$, the law of $u_{n}(t, x)$ is absolutely continuous with respect to the Lebesgue measure on $\mathbb{R}$.

Proof. It suffices to prove (3.15) in the particular case $\sigma=1$. Denote by $Q$ the process defined by

$$
D_{r, z} u_{n}(t, x)=G(t-r, x, z)+Q_{r, z}(t, x)
$$

and set

$$
\begin{aligned}
& I_{1}(t, x, \epsilon)=\int_{t-\epsilon}^{t} \int_{D} G^{2}(t-r, x, z) \mathrm{d} z \mathrm{~d} r \\
& I_{2}(t, x, \epsilon)=\int_{t-\epsilon}^{t} \int_{D} Q_{r, z}(t, x)^{2} \mathrm{~d} z \mathrm{~d} r .
\end{aligned}
$$

According to (3.21),

$$
\int_{0}^{t} \int_{D}\left|D_{r, z} u_{n}(t, x)\right|^{2} \mathrm{~d} z \mathrm{~d} r \geqslant \int_{t-\epsilon}^{t} \int_{D}\left|D_{r, z} u_{n}(t, x)\right|^{2} \mathrm{~d} z \mathrm{~d} r \geqslant \frac{1}{2} I_{1}(t, x, \epsilon)-I_{2}(t, x, \epsilon) .
$$

Let us find an upper estimate for $\mathrm{E}\left(I_{2}(t, x, \epsilon)\right) \leqslant 2\left(B_{1}(t, x, \epsilon)+B_{2}(t, x, \epsilon)\right)$, where

$$
\begin{aligned}
& B_{1}(t, x, \epsilon)=\int_{t-\epsilon}^{t} \int_{D} \mathrm{E}\left(\left|\int_{r}^{t} \int_{D} \Delta G(t-\theta, x, \eta) f_{n}^{\prime}\left(u_{n}(\theta, \eta)\right) D_{r, z} u_{n}(\theta, \eta) \mathrm{d} \eta \mathrm{d} \theta\right|^{2}\right) \mathrm{d} z \mathrm{~d} r \\
& B_{2}(t, x, \epsilon)=\int_{t-\epsilon}^{t} \int_{D} \mathrm{E}\left(\left|\int_{r}^{t} \int_{D} G(t-\theta, x, \eta) S_{n}(\theta, \eta) D_{r, z} u_{n}(\theta, \eta) W(\mathrm{~d} \eta, \mathrm{d} \theta)\right|^{2}\right) \mathrm{d} z \mathrm{~d} r .
\end{aligned}
$$

Schwarz's inequality applied with respect to the measure $|\Delta G(t-\theta, x, \eta)| \mathrm{d} \eta \mathrm{d} \theta$ and Fubini's theorem imply that

$$
\begin{aligned}
B_{1}(t, x, \epsilon) & \leqslant C_{n} \int_{t-\epsilon}^{t} \int_{D} \mathrm{E}\left(\int_{s}^{t} \int_{D}\left|\Delta G(t-\theta, x, \eta) \| D_{r, z} u_{n}(\theta, \eta)\right|^{2} \mathrm{~d} \eta \mathrm{d} \theta\right) \mathrm{d} z \mathrm{~d} r \\
& \leqslant C_{n} \int_{t-\epsilon}^{t} \int_{D}|\Delta G(t-\theta, x, \eta)| \mathrm{E}\left(\int_{t-\epsilon}^{\theta} \int_{D}\left|D_{r, z} u_{n}(\theta, \eta)\right|^{2} \mathrm{~d} z \mathrm{~d} r\right) \mathrm{d} \eta \mathrm{d} \theta .
\end{aligned}
$$

Using Lemma 3.3 with $t-\epsilon \leqslant \theta \leqslant t$ and (1.14), we deduce that 


$$
B_{1}(t, x, \epsilon) \leqslant C_{n} \epsilon^{1-d / 4} \int_{t-\epsilon}^{t} \int_{D}|\Delta G(t-\theta, x, \eta)| \mathrm{d} \eta \mathrm{d} \theta \leqslant C_{n} \epsilon^{3 / 2-d / 4} .
$$

For the second term, Burkholder's inequality implies that

$$
B_{2}(t, x, \epsilon) \leqslant C \int_{t-\epsilon}^{t} \int_{D} \mathrm{E}\left(\int_{s}^{t} \int_{D}|G(t-\theta, x, \eta)|^{2}\left|D_{r, z} u_{n}(\theta, \eta)\right|^{2} \mathrm{~d} \eta \mathrm{d} \theta\right) \mathrm{d} z \mathrm{~d} r .
$$

Computations similar to that for $B_{1}$ imply that

$$
B_{2}(t, x, \epsilon) \leqslant C \epsilon^{2-d / 4} \text {, }
$$

so that

$$
\mathrm{E}\left(I_{2}(t, x, \epsilon)\right) \leqslant C_{n} \epsilon^{(3 / 2-d / 4) \wedge(2-d / 4)} .
$$

We now need to find lower estimates for $I_{1}$. For this, we use the exact expression of the Green function $G$ given by (1.5), which yields

$$
I_{1}(t, x, \epsilon)=\int_{t-\epsilon}^{t} \int_{D}\left[\sum_{k \in \mathbb{N}^{d}} \varepsilon_{k}(x) \varepsilon_{k}(y) \exp \left(-\lambda_{k}^{2}(t-s)\right)\right]^{2} \mathrm{~d} y \mathrm{~d} s .
$$

Since the sequence $\left(\varepsilon_{k}\right)_{k \in \mathbb{N}^{d}}$ is an orthonormal basis for $L^{2}(D)$,

$$
\begin{aligned}
I_{1}(t, x, \epsilon) & =\int_{t-\epsilon}^{t}\left[\sum_{k \in \mathbb{N} d} \varepsilon_{k}^{2}(x) \exp \left(-2 \lambda_{k}^{2}(t-s)\right)\right] \mathrm{d} s . \\
& =\sum_{k \in \mathbb{N}^{d, *}} \varepsilon_{k}^{2}(x) \frac{1}{2 \lambda_{k}^{2}}\left[1-\exp \left(-2 \lambda_{k}^{2} \epsilon\right)\right]+C \epsilon .
\end{aligned}
$$

The series is well defined because $\sum_{k \in \mathbb{N} d, *} 1 / \lambda_{k}^{2}<\infty$. Let us choose $\left.x_{0}=\left(x_{\mathrm{i}}\right)_{i \in[1, d]} \in\right] 0, \pi\left[{ }^{d}\right.$. According to (1.4),

$$
I_{1}(t, x, \epsilon) \geqslant C \sum_{k \in\left(\mathbb{N}^{*}\right)^{d}} \Pi_{1 \leqslant i \leqslant d} \cos ^{2}\left(k_{i} x_{i}\right) \frac{1}{\left(\sum_{i=1}^{d} k_{i}^{2}\right)^{2}}\left[1-\exp \left(-2\left(\sum_{i=1}^{d} k_{i}^{2}\right)^{2} \epsilon\right)\right] .
$$

Given $\alpha$ in ]0, $x_{1} \wedge \ldots \wedge x_{d} \wedge \pi / 2[$, we observe the following:

Remark 3.5. If $k \in \mathbb{N}^{*}, x \in D$ are such that $\left.k x \in\right] \pi / 2-\alpha / 2, \pi / 2+\alpha / 2[(\bmod \pi)$, then

$$
(k+1) x \notin] \frac{\pi}{2}-\frac{\alpha}{2}, \frac{\pi}{2}+\frac{\alpha}{2}[(\bmod \pi) .
$$

The intervals $] \pi / 2-\alpha / 2, \pi / 2+\alpha / 2[(\bmod \pi)$ correspond to small values of the cosine function; in fact there exists $\beta>0$ such that $\cos ^{2}(x)>\beta$ if $\left.x \notin\right] \pi / 2-\alpha / 2$, $\pi / 2+\alpha / 2[(\bmod \pi)$. Using Remark 3.5, we obtain the following lower estimate, skipping every other term: 


$$
\begin{aligned}
I_{1}\left(t, x_{0}, \epsilon\right) & \geqslant C \beta^{2} \sum_{k \in\left(\mathbb{N}^{*}\right)^{d}}\left[\sum_{i=1}^{d}\left(1+2 k_{i}\right)^{2}\right]^{-2}\left[1-\exp \left(-2 \epsilon\left[\sum_{i=1}^{d}\left(2 k_{i}\right)^{2}\right]^{2}\right)\right] \\
& \geqslant C \beta^{2} \sum_{k \in\left(\mathbb{N}^{*}\right)^{d}}\left[\sum_{i=1}^{d} k_{i}^{2}\right]^{-2}\left(1-\exp \left(-8 \epsilon\left[\sum_{i=1}^{d} k_{i}^{2}\right]^{2}\right)\right) .
\end{aligned}
$$

Take $M>0$ small enough $(M<2)$ such that for $0<x<M$ we have $1-\exp (-x) \geqslant x / 2$; then since the number of points with integer coordinate in a circle (or sphere) or radius $r$ is dominated by $\mathrm{Cr}^{d}$,

$$
\begin{aligned}
I_{1}\left(t, x_{0}, \epsilon\right) & \geqslant C \beta^{2} \sum_{k \in\left(\mathbb{N}^{*}\right)^{d}, 8 \epsilon\left(\sum_{i=1}^{d} k_{i}^{2}\right)^{2}<M}\left[\sum_{i=1}^{d} k_{i}^{2}\right]^{-2}\left(1-\exp \left(-8 \epsilon\left[\sum_{i=1}^{d} k_{i}^{2}\right]^{2}\right)\right) \\
& \geqslant C \beta^{2} \sum_{k \in\left(\mathbb{N}^{*}\right)^{d}, 8 \epsilon\left(\sum_{i=1}^{d} k_{i}^{2}\right)^{2}<M} \epsilon \\
& \geqslant C \beta^{2} \epsilon\left(\frac{M}{8 \epsilon}\right)^{d / 4} \geqslant C C_{1} \beta^{2} \epsilon^{1-d / 4} .
\end{aligned}
$$

Inequalities (3.22), (3.24) and (3.25) yield

$$
\begin{aligned}
P\left(\int_{0}^{T} \int_{D}\left|D_{s, y} u_{n}(t, x)\right|^{2} \mathrm{~d} y \mathrm{~d} s>0\right) & \geqslant \sup _{0<\epsilon \leqslant \epsilon_{0}} P\left(\frac{1}{2} I_{1}(t, x, \epsilon)-I_{2}(t, x, \epsilon)>0\right) \\
& \geqslant \sup _{0<\epsilon \leqslant \epsilon_{0}} P\left(I_{2}(t, x, \epsilon)<C \frac{1}{2}^{1} \epsilon^{1-d / 4}\right) \\
& \geqslant 1-\inf _{0<\epsilon \leqslant \epsilon_{0}}\left\{\mathrm{E}\left(I_{2}(t, x, \epsilon)\right) \frac{2}{\epsilon^{1-d / 4} C}\right\} \\
& \geqslant 1-\inf _{0<\epsilon \leqslant \epsilon_{0}} C_{n} \epsilon^{1 / 2 \wedge(1-d / 4)} \\
& \geqslant 1 .
\end{aligned}
$$

This concludes the proof of Lemma 3.4.

\subsection{Proof of Theorem 1.5}

Let us now extend Lemma 3.4 to functions $\sigma$ which do not vanish. Let us denote by $v_{s, y}^{n}$ the solution to the $\operatorname{SPDE}$ (3.3) with $\sigma=1$, that is, for $t \geqslant s$, 


$$
\begin{aligned}
v_{s, y}^{n}(t, x)= & G(t-s, x, y)+\int_{s}^{t} \int_{D} \Delta G(t-\theta, x, \eta) f_{n}^{\prime}\left(u_{n}(\theta, \eta)\right) v_{s, y}^{n}(\theta, \eta) \mathrm{d} \eta \mathrm{d} \theta \\
& +\int_{s}^{t} \int_{D} G(t-\theta, x, \eta) S_{n}(\theta, \eta) v_{s, y}^{n}(\theta, \eta) W(\mathrm{~d} \eta, \mathrm{d} \theta),
\end{aligned}
$$

and $v_{s, y}^{n}(t, x)=0$ if $t<s$. Because of the uniqueness of the solution to (3.3),

$$
v_{s, y}^{n}(t, x) \sigma\left(u_{n}(s, y)\right)=D_{s, y} u_{n}(t, x)
$$

Also, because $\sigma$ does not vanish,

$$
\begin{aligned}
P\left(\left.\int_{0}^{t} \int_{D}|D| v_{s, y} u_{n}(t, x)\right|^{2} \mathrm{~d} y \mathrm{~d} s>0\right) & =P\left(\int_{0}^{t} \int_{D}\left|v_{s, y}^{n}(t, x) \sigma\left(u_{n}(s, y)\right)\right|^{2} \mathrm{~d} y \mathrm{~d} s>0\right) \\
& =P\left(\int_{0}^{t} \int_{D} 1_{\left\{\left|v_{s, y}^{n}(t, x) \sigma\left(u_{n}(s, y)\right)\right|^{2}>0\right\}} \mathrm{d} y \mathrm{~d} s>0\right) \\
& =P\left(\int_{0}^{t} \int_{D} 1_{\left\{\left|v_{s, y}^{n}(t, x)\right|^{2}>0\right\}} \mathrm{d} y \mathrm{~d} s>0\right) \\
& =P\left(\int_{0}^{t} \int_{D}\left|v_{s, y}^{n}(t, x)\right|^{2} \mathrm{~d} y \mathrm{~d} s>0\right) \\
& =1 .
\end{aligned}
$$

This proves (3.15), which implies that the law of $u_{n}(t, x)$ is absolutely continuous, and the localization $u_{\mid \Omega_{n}}=u_{n}$ yields Theorem 1.5.

\section{Appendix: Proof of Lemma 1.2}

We use a theorem from Eidelman and Ivasisen (1970), which gives a similar result for smooth domains. The difficulty is in the 'corners' of $D$, that is, the points $x$ at least two of whose coordinates belong to $\{0, \pi\}$, and where the boundary of $D$ is not smooth. Let us denote by $D^{\bullet}$ the parallelepiped $D$ without its 'corners'.

Let $D_{n}$ be an increasing sequence of smooth convex domains included in $D$ such that the intervals $\left\{x_{i} \in[1 / n, \pi-1 / n]\right\}$ of $\partial D$ belongs to $\partial D_{n}$. Denote by $G_{n}$ the Green function associated with the operator $\partial / \partial t+\Delta^{2}$ on $D_{n}$ with the Neumann boundary conditions. According to Eidelman and Ivasisen (1970, Theorem 1.1), and the first chapter of Eidelman and Zhitarashu (1998), we conclude that this parabolic system in the sense of Petrovskii is well defined and that the following inequalities hold for $x, y \in D_{n}, \alpha \leqslant 1,|\beta| \leqslant 4$ with constants which do not depend on $n$ : 


$$
\begin{gathered}
\left|G_{n}(t-s, x, y)\right| \leqslant \frac{C}{|t-s|^{d / 4}} \exp \left(-\frac{c|x-y|^{4 / 3}}{|t-s|^{1 / 3}}\right), \\
\left|D_{t}^{\alpha} D_{x}^{\beta} G_{n}(t-s, x, y)\right| \leqslant \frac{C}{|t-s|^{(d+4 \alpha+|\beta|) / 4}} \exp \left(-\frac{c|x-y|^{4 / 3}}{|t-s|^{1 / 3}}\right) .
\end{gathered}
$$

If $|\beta| \leqslant 3, D_{x}^{\beta} G_{n}$ is integrable; moreover, for $0 \leqslant t<t^{\prime} \leqslant T$,

$$
\int_{t}^{t^{\prime}} \int_{\mathbb{R}^{d}} D_{x}^{\beta} G_{n}(t-s, x, y) \mathrm{d} y \mathrm{~d} s \leqslant C\left|t-t^{\prime}\right|^{1-d / 4} .
$$

Let $\phi$ be a $C^{\infty}$ function on $[0, T] \times D$; for $(t, x) \in[0, T] \times D_{n}$, set

$$
w_{n}(t, x)=\int_{0}^{t} \int_{D_{n}} G_{n}(t-s, x, y) \phi(s, y) \mathrm{d} y \mathrm{~d} s .
$$

The function $w_{n}$ satisfies

$$
\frac{\partial w_{n}}{\partial t}(t, x)+\Delta^{2} w_{n}(t, x)=\phi(t, x)
$$

on $D_{n}$ with the homogeneous Neumann boundary conditions on $\partial D_{n}$. We first prove the convergence of $\left(w_{n}\right)$ on $[0, T] \times D^{\bullet}$.

Lemma A.1. For each $k>0$, the sequence $\left(w_{n}\right)_{n \geqslant k}$ is relatively compact in $\mathscr{C}\left([0, T] \times D_{k}\right)$. We can extract a subsequence $\tilde{w}_{n}$ such that its time derivative converges uniformly on each interval $[\epsilon, T] \times D_{k}$, for $\epsilon>0$, and such that its space derivative of order $\alpha$, with $|\alpha| \leqslant 3$, converges uniformly on $[0, T] \times D_{k}$. Let us denote by $\bar{w}_{k}$ the limit of $\tilde{w}_{n}$ on $[0, T] \times D_{k}$; there exists a function $w$ defined on $D^{\bullet}$ such that $\left.w\right|_{D_{k}}=\left.\bar{w}_{n}\right|_{D_{k}}$.

Proof. According to the Arzelà-Ascoli theorem, we have to prove that the sequence is bounded and equicontinuous. Inequalities (A.1) and (1.14) imply the uniform boundedness of the sequence.

Equicontinuity in time and in space are proved by different arguments, since $w_{n}$ is a convolution in time. Let $0 \leqslant t<t^{\prime} \leqslant T, x \in D_{k}$; then

$$
\begin{aligned}
w_{n}\left(t^{\prime}, x\right)-w_{n}(t, x)= & \int_{0}^{t} \int_{D_{n}} G_{n}(s, x, y)\left[\phi\left(t^{\prime}-s, y\right)-\phi(t-s, y)\right] \mathrm{d} y \mathrm{~d} s \\
& +\int_{t}^{t^{\prime}} \int_{D_{n}} G_{n}\left(t^{\prime}-s, x, y\right) \phi(s, y) \mathrm{d} y \mathrm{~d} s .
\end{aligned}
$$

The function $\phi$ is uniformly continuous on $[0, T] \times D_{k}$, and the upper estimate (A.1) of $G_{n}$ is independent of $n$; this implies that the first term on the right-hand side of (A.5) can be made less than $\epsilon$ for $t^{\prime}-t$ small enough uniformly in $x \in D_{k}$. Furthermore, using (A.1) and (1.14), we have 


$$
\left|\int_{t}^{t^{\prime}} \int_{D_{n}} G_{n}\left(t^{\prime}-s, x, y\right) \phi(s, y) \mathrm{d} y \mathrm{~d} s\right| \leqslant C\|\phi\|_{\infty}\left|t-t^{\prime}\right| ;
$$

this implies the equicontinuity of $w_{n}$ in the time variable (uniformly in the space variable). To study the space increment, let $x, x^{\prime} \in D_{k}$; then

$$
w_{n}(t, x)-w_{n}\left(t, x^{\prime}\right)=\int_{0}^{t} \int_{D_{n}}\left[G_{n}(t-s, x, y)-G_{n}\left(t-s, x^{\prime}, y\right)\right] \phi(s, y) \mathrm{d} y \mathrm{~d} s .
$$

Using (A.2) for the first-order partial derivatives with respect to the space variable and the Taylor formula, we deduce, by a computation similar to that made to prove (2.41) and (2.42), that

$$
\left|w_{n}(t, x)-w_{n}\left(t, x^{\prime}\right)\right| \leqslant C\left|x-x^{\prime}\right| \int_{0}^{t}|t-s|^{-1 / 4}\|\phi\|_{\infty} \mathrm{d} s .
$$

This yields the equicontinuity in the space variable in $D_{k}$, uniformly in the time variable. The sequence $w_{n}$ admits a subsequence $\left(\tilde{w}_{n}\right)_{n \geqslant k}$ which converges to $\bar{w}_{k}$ in $C\left([0, T] \times D_{k}\right)$. For the time derivative, we proceed in a similar way. We observe that

$$
\frac{\partial w_{n}}{\partial t}(t, x)=\int_{0}^{t} \int_{D_{n}} G_{n}(s, x, y) \phi_{t}^{\prime}(t-s, y) \mathrm{d} y \mathrm{~d} s+\int_{D_{n}} G_{n}(t, x, y) \phi(0, y) \mathrm{d} y .
$$

We have to prove equicontinuity in space and time variables and the boundedness of the sequence. The first term can be studied as $w_{n}(t, x), \phi$ being replaced by $\phi_{t}^{\prime}$. Because there is only a space integral, we prove equicontinuity and boundedness for the second term on $[\epsilon, T] \times D_{k}$. For the space increment, take $t, t^{\prime} \in[\epsilon, T]$; then

$$
\begin{aligned}
& \left|\int_{D_{n}}\left[G_{n}(t, x, y)-G_{n}\left(t^{\prime}, x, y\right)\right] \phi(0, y) \mathrm{d} y\right| \\
& \quad \leqslant\left|t-t^{\prime}\right| \int_{D} \int_{0}^{1} \frac{C}{\left(\theta t+(1-\theta) t^{\prime}\right)^{(d+4) / 4}} \exp \left(\frac{-c|x-y|^{4 / 3}}{\left(\theta t+(1-\theta) t^{\prime}\right)^{1 / 3}}\right) \mathrm{d} \theta \mathrm{d} y,
\end{aligned}
$$

Using (1.14), we conclude that the second term is equicontinuous in the time variable on $[\epsilon, T]$, uniformly in the space variable. We proceed in a similar way for the equicontinuity in the space variable. We can extract a further subsequence such that the time derivative converges too on $[\epsilon, T] \times D_{k}$ for $\epsilon>0$.

For the space derivative, let $\beta \in \mathbb{N}^{d}$ be such that $|\beta| \leqslant 3$; then we have

$$
D_{x}^{\beta} w_{n}(t, x)=\int_{0}^{t} \int_{D_{n}} D_{x}^{\beta} G_{n}(t-s, x, y) \phi(s, y) \mathrm{d} y \mathrm{~d} s .
$$

Uniform boundedness is a straightforward consequence of (A.2) and (1.14); the time increment of $D_{x}^{\beta} w_{n}$ can be studied like that of $w_{n}$. For the space increment, let $\left.\lambda \in\right] 0,1[$, apply Taylor's formula, (A.2) and (1.14) and proceed as in the proof of (2.41) and (2.42); this yields, for $\lambda \in[0,1]$, 


$$
\left|D_{x}^{\beta} w_{n}(t, x)-D_{x}^{\beta} w_{n}\left(t, x^{\prime}\right)\right| \leqslant\left|x-x^{\prime}\right|^{\lambda} \int_{0}^{t}|t-s|^{-(\beta+\lambda) / 4}\|\phi\|_{\infty} \mathrm{d} s
$$

We see that it suffices to take $\lambda$ such that $\beta+\lambda<4$. To obtain the equicontinuity of the derivatives of $w_{n}$ of order less than 3 in the space variable, we can finally extract a further sequence such that all the partial derivatives of order less than 3 with respect to the space variable converge on $[0, T] \times D_{k}$. The fact that there exists a function $w$ defined on $[0, T] \times D^{\bullet}$ comes from the fact that the values of the sequences do not depend on $k$. The function $w$ also has a time derivative and space derivative of order up to 3 , because the corresponding derivatives of $\left(w_{n}\right)$ converge uniformly on $] \epsilon, T[\times D$.

Since $\tilde{w}_{n}$ is a solution to (A.4), the sequence $\Delta^{2} \tilde{w}_{n}$ restricted to $D_{k}$ is uniformly convergent hence it also admits a limit point $w$ which satisfies the PDE

$$
\frac{\partial}{\partial t} w(t, x)=-\Delta^{2} w(t, x)+\phi(t, x)
$$

on $D^{\bullet}$. Furthermore, $w$ satisfies the Neumann boundary conditions because the function $w_{n}$ satisfies such conditions on the boundary of $D_{n}$, and we have constructed $D_{n}$ so that $\partial D_{n} \backslash \partial D$ increases to $\partial D \backslash\{0, \pi\}^{d}$ as $n \rightarrow \infty$. The initial condition is again $w(0, \cdot)=0$. Since (A.6) has a unique solution with homogeneous Neumann's condition, we deduce that

$$
w(t, x)=\int_{0}^{t} \int_{D} G(t-s, x, y) f(s, y) \mathrm{d} y \mathrm{~d} s,
$$

where $G$ is the Green kernel given by (1.5). The sequence $\left(G_{n}(\cdot, x, \cdot)\right)_{n \geqslant k}$ also converges weakly on $[0, t] \times D_{k}$ for every $\left.\left.t \in\right] 0, T\right]$ and every $x \in D_{k}$.

We need to extend upper estimates (A.1) and (A.2) from $G_{n}$ to $G$. Let $s_{0}, y_{0}$, $\left.x_{0} \in\right] 0, T\left[\times(] 0, \pi\left[{ }^{d}\right)^{2}\right.$; there exist $k$ such that $y_{0}$ and $x_{0}$ belong to $D_{k-1} \backslash \partial D_{k-1}$. Let $\chi$ be a positive $C^{\infty}$ function on $\mathbb{R} \times \mathbb{R}^{d}$ with compact support included in $[-1,1]^{d+1}$, such that $\int_{[-1,1]^{d+1}} \chi(x) \mathrm{d} x=1$, and set

$$
\chi_{s_{0}, y_{0}, \epsilon}(s, x)=\epsilon^{-(d+1)} \chi\left(\frac{s-s_{0}}{\epsilon}, \frac{x-y_{0}}{\epsilon}\right)
$$

then the sequence $\chi_{s_{0}, y_{0}, \epsilon}$ converges weakly to $\delta_{s_{0}, y_{0}}$ in $\mathscr{D}\left([0, T] \times D_{k}\right)$. For $\epsilon$ small enough, the support of $\chi_{s_{0}, y_{0}, \epsilon}$ is included in $\left[0, t_{0}\right] \times D_{k}$; hence for fixed $t_{0}$, we have

$$
\begin{aligned}
\int_{0}^{t_{0}} \int_{D} G\left(t_{0}-s, x_{0}, y\right) \chi_{s_{0}, y_{0}, \epsilon}(s, y) \mathrm{d} y \mathrm{~d} s & =\int_{0}^{t_{0}} \int_{D_{k}} G\left(t_{0}-s, x_{0}, y\right) \chi_{s_{0}, y_{0}, \epsilon}(s, y) \mathrm{d} y \mathrm{~d} s \\
& =\lim _{n \rightarrow \infty} \int_{0}^{t_{0}} \int_{D_{k}} G_{n}\left(t_{0}-s, x_{0}, y\right) \chi_{s_{0}, y_{0}, \epsilon}(s, y) \mathrm{d} y \mathrm{~d} s
\end{aligned}
$$

The function $\chi$ is positive, and (A.1) implies that 


$$
\begin{aligned}
& \left|\int_{0}^{t_{0}} \int_{D} G\left(t_{0}-s, x_{0}, y\right) \chi_{s_{0}, y_{0}, \epsilon}(s, y) \mathrm{d} y \mathrm{~d} s\right| \\
& \quad \leq C \int_{0}^{t_{0}} \int_{D_{k}} \frac{1}{\left|t_{0}-s\right|^{d / 4}} \exp \left(-\frac{c\left|x_{0}-y\right|^{4 / 3}}{\left|t_{0}-s\right|^{1 / 3}}\right) \chi_{s_{0}, y_{0}, \epsilon}(s, y) \mathrm{d} y \mathrm{~d} s .
\end{aligned}
$$

On the other hand, we know that the kernel $G$ is defined by (1.5); hence $G$ is a continuous function on $] 0, T] \times D^{2}$, so that

$$
\lim _{\epsilon \rightarrow 0} \int_{0}^{t_{0}} \int_{D} G\left(t_{0}-s, x_{0}, y\right) \chi_{s_{0}, y_{0}, \epsilon}(s, y) \mathrm{d} y \mathrm{~d} s=G\left(t_{0}-s_{0}, x_{0}, y_{0}\right) .
$$

Therefore, as $\epsilon$ tends to $0,($ A.7) yields

$$
\left|G\left(t_{0}-s_{0}, x_{0}, y_{0}\right)\right| \leqslant \frac{C}{\left|t_{0}-s_{0}\right|^{d / 4}} \exp \left(-\frac{c\left|x_{0}-y_{0}\right|^{4 / 3}}{\left|t_{0}-s_{0}\right|^{1 / 3}}\right) .
$$

We observe that for $|\alpha| \leqslant 3$,

$$
\partial_{x}^{\alpha} w(t, x)=\int_{0}^{t} \int_{D} \partial_{x}^{|\alpha|} G(t-s, x, y) f(s, y) \mathrm{d} y \mathrm{~d} s .
$$

Because $\partial_{x}^{\alpha} \tilde{w}_{n}$ converges to $\partial_{x}^{\alpha} w ;\left(\partial_{x}^{\alpha} G_{n}(\cdot, x, \cdot)\right)_{n \geqslant k}$ converges weakly to $G(\cdot, x, \cdot)$ on $[0, T] \times D_{k}$. Arguments similar to the preceding ones imply that, for $|\alpha| \leqslant 3$,

$$
\left|\partial_{x}^{\alpha} G\left(t_{0}-s_{0}, x_{0}, y_{0}\right)\right| \leqslant \frac{C}{\left|t_{0}-s_{0}\right|^{(d+|\alpha|) / 4}} \exp \left(-\frac{c\left|x_{0}-y_{0}\right|^{4 / 3}}{\left|t_{0}-s_{0}\right|^{1 / 3}}\right)
$$

and

$$
\left|\Delta^{2} G\left(t_{0}-s_{0}, x_{0}, y_{0}\right)\right| \leqslant \frac{C}{\left|t_{0}-s_{0}\right|^{(s+4) / 4}} \exp \left(-\frac{c\left|x_{0}-y_{0}\right|^{4 / 3}}{\left|t_{0}-s_{0}\right|^{1 / 3}}\right) .
$$

Because $G$ is the Green kernel associated with the operator $\partial / \partial t+\Delta^{2}, G$ satisfies

$$
\frac{\partial}{\partial t} G=-\Delta^{2} G
$$

if $t>0, x, y \in D$, and the estimate (1.8) can be deduced from (A.8); this concludes the proof of Lemma 1.2.

\section{Acknowledgements}

I would like to thank A. Millet for her never-ending help in the preparation of this paper. 


\section{References}

Adams, R.A. (1975) Sobolev Spaces. New York: Academic Press.

Bally, V., Millet, A. and Sanz-Solé, M. (1995) Approximation and support theorem in Hölder norm for parabolic stochastic partial differential equations. Ann. Probab., 23, 178-222.

Cahn, J.W. and Hilliard, J.E. (1958) Free energy for a nonuniform system I. Interfacial free energy. J. Chem. Phys., 2, 258-267.

Da Prato, G. and Debussche, A. (1996) Stochastic Cahn-Hilliard equation. Nonlinear Anal., 26, 241-263.

Da Prato, G. and Gatarek, D. (1995) Stochastic Burgers equation with correlated noise. Stochastics Stochastics Rep., 52, 29-41.

Da Prato, G. and Zabczyk, J. (1992) Stochastic Equations in Infinite Dimensions, Encyclopedia of Mathematics and its Applications, Vol. 44. Cambridge: Cambridge University University Press.

Debussche, A. and Dettori, L. (1995) On the Cahn-Hilliard equation with a logarithmic free energy. Nonlinear Anal., 24, 1497-1514.

Eidelman, S.D. and Ivasisen, N.V. (1970) Investigation of the Green matrix for a homogeneous parabolic boundary value problem. Trans. Moscow Math. Soc., 23, 179-242.

Eidelman, S.D. and Zhitarashu, N.V. (1998) Parabolic Boundary Value Problems. Basel: Birkhäuser.

Garsia, A. (1972) Continuity properties of Gaussian processes with multi-dimensional time parameter. In L. LeCam, J. Neyman and E.L. Scott (eds), Proceedings of the Sixth Berkeley Symposium on Mathematical Statistics and Probability, Vol. 2, pp. 369-374. Berkeley: University of California Press.

Gyöngy, I. (1998) Existence and uniqueness results for semilinear stochastic partial differential equations. Stochastic Process. Appl., 73, 271-299.

Kunita, H. (1984) Stochastic differential equations and stochastic flows of diffeomorphisms. In P.L. Hennequin (ed.), École d'été de Probabilités de St-Flour XII, 1982, Lecture Notes in Math. 1097, pp. 143-303. Berlin: Springer-Verlag.

Lanjri Zaidi, N. and Nualart, D. (1999) Burgers equation driven by a space-time white noise: absolute continuity of the solution. Stochastics Stochastics Rep., 66, 273-292.

Morien, P.-L. (1999) On the density for the solution of a Burgers-type SPDE. Ann. Inst. H. Poincaré Probab. Statist., 35, 459-482.

Nualart, D. (1995) The Malliavin Calculus and Related Topics. Berlin: Springer-Verlag.

Novick-Cohen, A. and Segel, L.A. (1984) Nonlinear aspects of the Cahn-Hilliard equation. Physica $D, 10,277-298$.

Walsh, J.B. (1986) An introduction to stochastic partial differential equations. In P.L. Hennequin (ed.), École d'Été de Probabilités de St-Flour XIV, 1984, Lecture Notes in Math. 1180, pp. 265-439. Berlin: Springer-Verlag.

Received December 1999 and revised February 2001 